\title{
Rare ou abondante, l'eau précieuse. En France, l'exemple de la Brenne des étangs
}

\section{Geneviève Bédoucha}

\section{(2) OpenEdition}

1 Journals

Édition électronique

URL : http://journals.openedition.org/aa/1139

DOI : $10.4000 / a a .1139$

ISSN : 2357-738X

Éditeur

Programa de Pós-Graduação em Antropologia Social (UnB)

\section{Édition imprimée}

Date de publication : 1 décembre 2011

Pagination : 127-167

ISSN : 0102-4302

\section{Référence électronique}

Geneviève Bédoucha, «Rare ou abondante, l'eau précieuse. En France, l'exemple de la Brenne des étangs », Anuário Antropológico [En ligne], v.36 n.1 | 2011, mis en ligne le 24 novembre 2015, consulté le 28 avril 2021. URL : http://journals.openedition.org/aa/1139; DOI : https://doi.org/10.4000/aa.1139

\section{(c) $(1)(9)$}

Anuário Antropológico is licensed under a Creative Commons Atribuição-Uso Não-Comercial-Proibição de realização de Obras Derivadas 4.0 International. 


\section{Rare ou abondante, l'eau précieuse. En France, l'exemple de la Brenne des étangs}

Geneviève Bédoucha

CNRS, Paris

Avant même que n'ait été véritablement entreprise l'analyse en Brenne d'un système de gestion des eaux d'étangs voués à l'exploitation piscicole depuis l'époque monastique et qu'en ait été mesurée l'importance, surgissaient différentes questions stimulantes.

En cette région du cœur de la France où se trouvent d'innombrables étangs, classée zone humide d'importance internationale, le lien entre gestion de l'eau et société serait-il aussi signifiant qu'il est avéré en zone aride ou semi-aride ? En d'autres termes, la gestion de l'eau pouvait-elle y structurer le social, et la société se projeter dans l'organisation hydraulique, comme cela a pu être observé à maintes reprises en différents lieux du monde ${ }^{1}$ ? Ligne fructueuse de déchiffrement du social là où elle est essentielle à la survie d'une société, l'eau peut-elle permettre d'accéder au social là où l'on ne saurait prétendre qu'elle soit condition de survie, ou encore là où elle existerait même en abondance ?

Le paradoxe d'une gestion rigoureuse des eaux, alors que l'on semblerait bien être en situation d'abondance, a été souligné dans des travaux récents menés au sein d'aires géographiques et culturelles très diverses, et portant sur des sociétés où se pratique une agriculture irriguée. Ces travaux, dont j'interprèterai ici en partie les résultats, amènent à discuter de la notion même d'abondance, dont on verra qu'elle peut être toute relative, et dès lors de la perception que peuvent avoir à différents moments de leur histoire les différentes sociétés de la notion de rareté, du sens qu'elles lui donnent.

Que la rareté de l'eau même en région aride n'exclut en rien une déperdition objective d'eau, seule évaluée par des scientifiques extérieurs, est d'ailleurs hautement significatif de la perception que peuvent avoir les sociétés de la rareté. Aussi, observer des déperditions d'eau, des « gaspillages », dans une société pratiquant l'irrigation dans un contexte d'eau abondante, ne saurait non plus être la preuve du caractère non précieux de l'eau aux yeux des hommes. 


\section{Paradoxes apparents}

Le choix, délibéré, d'une société située au Portugal, dans le canton de Melgaço où l'eau existe véritablement en abondance, a été celui de Fabienne Wateau (2002) pour laquelle il s'agissait clairement « d'écarter la question de la rareté de l'eau », l'eau qui, dans la vision économiste, et parce qu'elle suscite la convoitise, «légitime les conflits ». Abondance flagrante de l'eau avec toutefois la mise en œuvre, exclusivement durant la saison d'été — même s'il repose sur des infrastructures simples — d'un système d'irrigation complexe dans la diversité même des types de partage, les modes de comptage minutieux des droits d'eau, la logique de répartition des eaux.

L'aménagement de ce système, qui — cela a toute son importance, ne concerne pas l'ensemble de la population —, date du xviie siècle. La richesse en eau provenant de sources et de ruisseaux des montagnes proches avait alors permis de diversifier et d'intensifier la production agricole, avec notamment l'introduction de la culture du maïs, grande consommatrice d'eau mais aussi celle du haricot et de la courge. L'inauguration d'une «irrigation intensive », qui a vu se multiplier forages de galeries drainantes et rigoles avec organisation de tours d'eau et un partage strictement réglementé, daterait de cette époque. Irrigation intensive par une « eau d'abondance » pour une production agricole accrue et diversifiée qui aurait entraîné un essor démographique notoire: telle se présente la situation à Melgaço.

Au Népal, dans la région d'Aslewacaur, alors que l'eau s'y trouve en quantité suffisante pour alimenter toutes les rizières, une distribution stricte en est pourtant observée (Aubriot 2004). L'organisation des tours d'eau connaît un ordonnancement rigoureux en quatre quartiers d'irrigation par canal - auxquels sont attribués des temps d'irrigation fixes —-, ainsi qu'une alternance des tours d'eau entre amont et aval, jour et nuit, qui garantit l'accès à l'eau dans une parfaite égalité des groupes lignagers. Le partage précis de l'eau y est d'autant plus contraignant que l'on a recours à l'horloge à eau ordinairement utilisée dans les milieux arides. Et l'on peut légitimement s'interroger dans un premier temps sur une telle minutie du partage de l'eau en général associée à la rareté de l'eau.

A contrario, dans une société oasienne située, elle, en zone aride, au Ladakh, région des hautes vallées de l'Himalaya, le partage de l'eau ne fait aucunement l'objet d'une réglementation précise (Labbal 2001). Discutons-en d'emblée le paradoxe. Ici, on peut avancer qu'elle ne s'avère pas nécessaire, car l'interdit de troubler l'ordre d'irrigation auquel veillent les maîtres de l'eau, chargés chaque mois des rituels honorant les divinités, relève du sacré; il est si profondément intégré qu'extrêmement rares sont les fauteurs de trouble. Un des mots-clefs de 
la pensée religieuse apparaît de façon récurrente dans les discours, qui renvoie à la fois à d'heureux auspices et à la notion d'interdépendance, celle des hommes, des dieux, des forces de la nature étroitement liés dans la recherche de la prospérité: « tous participent à un ordre du monde qui, s'il n'est pas préservé, laissera place au chaos aux malheurs et aux calamités. » (Labbal, 2001:196).

L'eau passe de champ en champ et le débit attribué correspond aux besoins précis de la parcelle selon les cultures pratiquées, évalués par les maîtres de l'eau. Autrefois entre les mains de fonctionnaires du roi qui se la transmettaient par héritage, la charge revient de nos jours par rotation aux membres de la communauté souvent réticents à l'accepter du fait des compétences fines qu'elle exige, de la lourdeur de la charge et de la responsabilité qu'elle suppose. Chaque parcelle a ainsi ce qu'il lui faut mais il est entendu que personne n'abusera, ou risquera de gaspiller l'eau. Personne n'y a intérêt, celui qui enfreint les règles tacites se verrait mis au ban de la société et les conséquences peuvent être graves pour l'ensemble de la communauté qui craint les représailles des divinités en colère.

Ainsi, nul besoin dans cette société, me semble-t-il, et malgré un contexte de rareté de l'eau, de recourir à un partage strict. Les valeurs profondément ancrées et partagées, éventuellement la pression de la communauté sur les individus, sont suffisamment fortes pour que soit respectée la discipline par l'ensemble de la communauté. Les divinités ont seules le véritable pouvoir sur l'eau comme sur l'ensemble des ressources du territoire, seules elles peuvent assurer à l'oasis l'eau suffisante pendant toute la saison.

L'abondance énoncée de l'eau à Aslewacaur ou à Melgaço trouve cependant dans les deux cas, ici très rapidement présentés, chaque fois d'évidentes limites qui selon moi pourraient amener à relativiser la notion d'abondance.

À Aslewacaur, le canal principal d'irrigation, creusé sur $6 \mathrm{kms}$ à flanc de montagne avant sa partition en trois canaux secondaires, est menacé par de fréquents glissements de terrain qui provoquent des interruptions brutales de l'alimentation en eau. Dans un contexte d'abondance, un risque bien réel de manque d'eau existe donc, pendant certaines périodes — précisément lorsqu'un apport d'eau est essentiel à la riziculture —, qui peut induire une tension sociale. Et les hommes ne l'ont pas négligé. C'est bien le risque de pénurie du fait de la rupture possible du canal qui aurait amené à une distribution extrêmement stricte, à un découpage du temps précis et rigide, chaque parcelle ayant un droit bien défini. L'ordre aurait été établi de façon à éviter tout conflit en cas de pénurie soudaine, il est fixe, et sans égard pour le manque d'eau provoqué l'irrigation se poursuit selon un ordre immuable, indifférent aux incidents. 
Les ayants droits lésés devront, eux, attendre le tour suivant. Aussi les règles de partage ont-elles pu être aménagées au sein d'un même lignage afin de diminuer l'écart entre deux tours.

L'utilisation de l'horloge à eau ne vient que conforter, accentuer ce caractère rigoureux et indiscutable du partage. Le recours à cette technique savante de mesure du temps ordinairement utilisée au Népal par les astrologues viendrait par sa référence spirituelle «marquer l'influence cosmique » sur la gestion d'ensemble (Aubriot, 2004:15). Il a dans cette société une portée symbolique qui aurait aidé à lever les réticences et à l'acceptation du système mis en place, non sans mal, par un seul homme.

Situation toute différente dans le cas portugais: l'extension au xvii ${ }^{\mathrm{e}}$ siècle à Melgaço de cultures très exigeantes en eau pourrait bien avoir rendu caduque la notion d'abondance durant la saison d'été. Fortement mobilisée, l'eau devient une ressource précieuse que l'on se répartit dès lors avec vigilance. Mais il y a là incontestablement un choix de la société melgacense, à un moment précis de son histoire, d'exercer une plus grande pression sur l'eau.

«Donnée naturelle ou question sociale »? L'interrogation, suscitée plus largement à propos de la pénurie d'eau, n'est pas nouvelle et se poursuit, approfondie par des géographes lors d'une publication collective. L'une des contributions (Jacob-Rousseau, 2005) analyse avec précision comment, dans le bassin de l'Ardèche du xix ${ }^{\mathrm{e}}$ siècle, on a ainsi pu passer d'une pénurie conjecturelle et saisonnière à une pénurie structurelle et chronique, du fait même de besoins accrus, et d'une forte augmentation de prélèvements, tant agricoles qu'industriels qui sont allés au-delà des ressources disponibles notamment durant la saison chaude. Naturels ou sociaux, les «facteurs limitants » ont été nombreux, et l'économie hydrique, qui aggravait les étiages, avait sa part dans la production de pénurie que différents aménagements hydrauliques relativement modestes sont venus ainsi atténuer.

Partout dans le monde lorsque le contexte est celui d'une pénurie d'eau, les sociétés ne manquent pas d'inventivité, de nombreux travaux d'ethnologues l'ont démontré, qui sont devenus essentiels aujourd'hui à une réflexion plus large sur le manque d'eau à grande échelle et la perception qu'ici ou là on peut en avoir.

S'appuyant sur les travaux de Latour (1993), Julie Trottier (2005:198) reprend à juste titre le terme « hybrid », pertinent pour qualifier une crise de l'eau qui ne saurait être exclusivement naturelle: «It includes a non-human object, water, as well as a complex array of values, intentions and expectations that lead a material situation to be defined as a crisis. » Mais différents travaux encore le démontrent ${ }^{2}$, le manque d'eau peut aussi résulter d'une décision, la rareté de 
l'eau peut en grande partie être le produit d'une construction sociale. Si l'on n’a jamais dû connaître une véritable crise de l'eau dans la région étudiée par F. Wateau, l'eau n'est-elle pas au xvii siècle devenue indispensable en été à l'irrigation de cultures nouvelles, et considérée suffisamment précieuse pour que les hommes aient estimé impératif d'élaborer un système d'irrigation destiné à en contrôler sévèrement la répartition?

Aujourd'hui, alors même que la production agricole ne présente plus le même intérêt, et que le nombre d'usagers est plus restreint, le système d'irrigation demeure dans toute sa complexité de détails foisonnante, avec les mêmes contraintes en saison d'été où une gestion rigoureuse d'une eau strictement rationnée continue de prévaloir: elle a été établie à un certain moment et bien, me semble-t-il, parce que l'eau est alors devenue un enjeu. L'irrigation, en permettant une production accrue, a amené une prospérité nouvelle, qui a permis à certaines familles de se développer plus largement et d'affirmer leur position. Elle reste nécessaire en saison d'été pour les « héritiers » qui n'entendent pas rompre avec l'ancestrale habitude des cultures irriguées. L'enjeu ainsi peut ensuite se transformer, changer de nature et si l'eau n'a plus la même importance économique, devenir par exemple, comme à Melgaço un enjeu exclusivement et fortement identitaire. Qualifiée aujourd'hui d'abondante, de abundância, ${ }^{3}$ destinée d'ailleurs à une production que l'on pourrait qualifier de superflue, l'eau d'irrigation à Melgaço est chargée d'une valeur précieuse entre toutes, dans cette société parce que signe de prestige et de distinction sociale : personne, parmi ceux que l'on nomme «les héritiers », et qui seuls possèdent l'eau durant la saison, ne songerait à se séparer de ses droits. Tous se retrouvent au moment du cycle d'irrigation inauguré chaque été, se montrant pointilleux sur les règles de distribution connues de tous, et dont tous d'ailleurs se réclament, et perpétuent une tradition à laquelle ils sont toujours attachés. Dans l'un des films tournés par F. Wateau (2008), un des «héritiers » évoque à propos du fonctionnement des mares « qui, sans aucun doute, dit-il, existent depuis qu'il y a des cultures », « un système primitif », «très ancien », des «partages originels». Avec une sorte de fatalisme car il n’a pas prise sur les choses, il énonce déjà, de façon significative, qu' « en principe elles existeront tant qu'il y aura les cultures », celles-là mêmes qui ont exigé la mise en place d'un système d'irrigation, et qui sont bien au fondement de leur privilège. Et peut-être faut-il entendre dans sa voix qui laisse percer impuissance désabusée et regret : tant que subsisteront des héritiers intéressés aux cultures. Car il ne doute pas qu' "on arrivera à un point où la terre cessera d'être cultivée et où les mares perdront leur raison d'être ».

Parce que, désormais, elle «participe de la construction identitaire des 
groupes » (Wateau, 2002:13), l'eau a cependant gardé un rôle capital dans cette société. Abondante certes, ${ }^{4}$ mais fortement mobilisée et dès lors précieuse. Précisément distribuée, et dès lors éminemment conflictuelle. Dans le climat agonistique général de cette société, l'eau, même abondante, n'échappe pas au conflit. Au contraire, elle y apparaît le lieu privilégié, et pour l'ethnologue attentive aux conflits et au défi un des moyens d'accès privilégié au social, comme dans de nombreux autres exemples de sociétés où l'eau pour une raison ou une autre a une place essentielle.

En Brenne dans le centre de la France ( $c f$. Fig. 1), ${ }^{5}$ on dénombre des milliers d'hectares en eau et des étangs par milliers, mais ici encore, alors que rien ne pouvait le laisser supposer a priori, l'analyse précise et en profondeur du système technique d'utilisation des eaux laisse apparaitre une gestion de l'eau comme d'une ressource précieuse (Bédoucha 2011). ${ }^{6}$ Cela sera démontré de façon plus détaillée : tout est pensé et fait dans le système d'exploitation piscicole des étangs pour perdre le moins d'eau possible, en gaspiller le moins possible. L'exercice est d'autant plus difficile qu'il n'existe aucune instance collective de gestion de l'eau. Le système d'ailleurs se révèle de ce fait d'une grande complexité de gestion, à l'évidence elle-même liée au caractère précieux de l'eau qu'il nous découvre.

Pas d'organisation centralisée de l'irrigation non plus dans le canton de Melgaço au Portugal, ni contrôle de l'ensemble du groupe d'ayants droits, mais il s'agit là d'une communauté paysanne, vivant et exploitant un terroir dans la continuité, et dans la continuité elle perpétue une tradition. Ce n'est pas le cas en Brenne, terre de grande propriété foncière où les étangs ont longtemps été la propriété exclusive de familles à la tête de grands domaines, où ces familles enfin, hormis quelques-unes d'entre elles, ancrées dans l'espace depuis un à deux siècles, n'ont cessé de se renouveler, et prisent toujours au plus haut point l'indépendance au sein de leur domaine. Indépendance illusoire, nous le verrons, car le lien effectif des étangs entre eux amène ou contraint les hommes à accepter un minimum de discipline dans l'intérêt collectif.

Précieuse dans la gestion des étangs pour les propriétaires qui continuent à privilégier la pisciculture mais à des fins qu'ils nous reste ici à explorer, l'eau l'est aussi éminemment pour le monde agricole alentour. Depuis des siècles, qu'elle fasse l'objet d'un droit lié aux terres riveraines ou d'une tolérance de la part des propriétaires d'étangs, elle est un appoint essentiel, souvent vital pour l'abreuvage des bêtes durant la saison sèche. Les pacages plus humides qui jouxtent l'eau des étangs, ont tout leur prix, comme la possibilité de faucher les joncs qui, longtemps, jusqu'après la deuxième guerre et faute de paille 
suffisante, étaient nécessaires aux litières des étables. En cela, les agriculteurs sont dépendants des propriétaires d'étangs qui se révèleront eux-mêmes dépendants à différents égards du monde paysan. Ainsi, autour des étangs, se sont noués des rapports étroits et durables.

\section{Une économie stricte de l'eau dans l'exploitation piscicole}

Un vaste système d'étangs solidaires

Par la nature de ses terres, par l'omniprésence anciennement attestée des eaux d'étangs et le fait que les eaux, au sein de groupes d'étangs reliés entre eux par un réseau complexe de fossés et de ruisseaux, rejoignent l'une ou l'autre rivière, Claise ou Creuse, qui enserrent le pays, la Brenne centrale forme sans conteste un ensemble bien particulier (cf. Fig. 2). Cette «pleine Brenne », à laquelle les hommes se réfèrent aussi si fréquemment, définirait de façon plus restreinte, plus condensée, le cœur même d'un pays, en ce qu'il a de plus singulier : ses étangs les plus vastes, les plus anciens, les plus prestigieux, qui en ponctuent et en marquent l'espace et gardent une forte prégnance symbolique dépassant largement ses frontières.

Le fonctionnement du système d'exploitation des eaux d'étangs est analysé non pas seulement sur un groupe particulier d'étangs, mais bien de proche en proche, dans sa totalité et sa cohésion à l'échelle de la région telle que la société la dessine elle-même, avec pour hypothèse que la gestion des eaux, dans la tension même qu'elle suppose, structure l'ensemble de la société. Un système dont le fonctionnement, paradoxalement dans une région de propriété privée et sans organisation communautaire, exigerait justement un contrôle — ou au moins une discipline - collectif.

Pour parler de la Brenne, les gens du pays aiment à en définir avant tout les limites, ils en visualisent immédiatement des frontières claires, précises, parfois invisibles à l'œil profane. Avec une sorte d'évidence, que ce soit dans une cuisine paysanne, dans le salon d'un château ou d'une demeure bourgeoise, elles sont fermement dessinées. Au sein de cet espace auquel s'identifie fortement la société locale, une réelle densité d'étangs existe : un très grand nombre, et les plus importants des plus de deux milliers d'étangs aujourd'hui dénombrés, ${ }^{7}$ s'y voient concentrés. La représentation cartographique qui les fait seuls apparaître offre au regard une extraordinaire constellation, plus chargée encore en son centre (cf. Fig. 3).

Des limites, le discours passe parfois instantanément à la nature particulière de la terre. Car leur dessin que tout le monde a à l'esprit, lui est lié, et l'on ne peut mieux définir le pays que par sa terre, dont progressivement les 
agriculteurs ont bien dû abandonner la culture au profit du seul élevage... De la terre, ils parlent avec véhémence tant ils tiennent à en faire comprendre la spécificité. En quelques mots concrets, et comme pour résumer et parler au mieux du pays, ils en déclinent les caractéristiques: «Du sable tout cru, le roc en dessous », " Ça d'terre, au-dessus: d'l'argile », ou encore, une sorte de « sorbet de sable et argile mêlés », et en dessous du grès, qui parfois affleure ou, en certains endroits, lorsqu'il a résisté à l'érosion, surgit en tertres, buttons larges de quelques dizaines de mètres où s'accrochent ajoncs et bruyères, dispersés sur la partie la plus haute de la Brenne. "Aussi vite sec que mouillé », «Quatre gouttes d'eau c'est mouillé, deux coups de soleil c'est grillé », «Dès qu'il fait chaud, ça grille», « Noyée ou brûlée, c’est ça, la Brenne ». Mouillé/grillé, noyé/ brûlé: d'une paire de mots, tout est dit. Les agriculteurs associent toujours les mots extrêmes ensemble, résumant ainsi de façon plus frappante leur propos.

Terre mouillée, trempée, ennoyée: l'eau est immédiatement présente dans le discours. Décrire la terre, c'est aussitôt en même temps parler de l'eau, des étangs et de leur densité. Les hommes évoquent une terre humide, tout de suite argileuse sous la mince couche arable, qui capture et retient l'eau mais sans l'absorber, une terre dont les moindres creux se transforment en «mouillères » car l'eau y stagne, une terre qui se défonce sous le poids des bêtes, et dans laquelle les tracteurs s'enlisent. À peine passée la Claise au nord, on fait la différence : «Ici on patine un peu, là-bas on se défonce pour un rien. »

On n’a cessé en Brenne d'évaluer le nombre des étangs et l'espace qu'ils occupent. Sans doute la forte présence des eaux dans le paysage a-t-elle toujours été si frappante qu'elle amenait au désir d'en avoir une mesure. L'obsession de compter les étangs traverse les siècles, à des moments différents de l'histoire et pour des causes diverses. Bien avant que l'État ait réellement besoin de les recenser pour intervenir, bien avant que l'on ait à s'alarmer de leur création " anarchique », déraisonnée, on dénombrait les étangs. C'est aujourd'hui un exercice nécessaire pour les organismes d'État, les particuliers s'y livrent aussi. Et le nombre d'étangs est toujours saisissant par l'extraordinaire densité qu'il révèle.

Bien sûr les chiffres varient, même dans des textes contemporains, selon l'espace pris en considération. Et ils ne cessent d'évoluer, car il reste difficile pour les organismes d'État de véritablement contrôler la création d'étangs de superficie inférieure à 3 hectares, et l'on n'est toujours pas en mesure de contrôler celle d'étangs de superficie inférieure à 1 hectare. Les hommes enfin ont toujours le moyen de ruser avec la loi. Mais qu'importe pour notre propos le nombre exact : retenons un phénomène large de création récente des étangs et 
ses conséquences dans la gestion de l'eau.

En « chaînes », en « chapelets », en « lignées », ces étangs s'agencent dans l'espace. Termes d'un usage courant dont on signalera juste ici la forte connotation symbolique: ${ }^{8}$ ils désignent des suites d'étangs qui se vident les uns dans les autres au sein d'un même bassin versant. Pour garder une certaine distance, le terme plus neutre de «lignes », qui décrit de façon tout aussi juste et imagée l'inscription des étangs dans l'espace, sera retenu.

Nombreux, ces étangs sont aussi très proches les uns des autres. Pour ceux qui y habitent, la particularité encore de la Brenne par rapport à d'autres régions d'étangs (comme la Sologne notamment, autre région du centre de la France), outre la densité des étangs et l'importance des surfaces en eau comparée à la surface des terres, c'est aussi cette très grande proximité des étangs les uns des autres, cette présence de l'eau partout dans les terres. Des étangs qui souvent même se jouxtent, ou que parfois une simple digue sépare. Exploitants, pisciculteurs ou gardes, les hommes d'étangs en général, ${ }^{9}$ parlent d'étangs «à la queue leu leu», « en cascade », qui « se bousculent» les uns les autres. Dans leur vocabulaire, dans le rythme des phrases, ils transmettent d'emblée ce « stress », terme récurrent lui aussi, lié à la période des pêches concentrées sur quelques mois, de la fin octobre à février-mars, et qui exige, pour que soit récupérée le plus d'eau possible, le vidage successif et planifié des étangs rendus solidaires les uns des autres par un réseau dense de fossés et de ruisseaux.

Les étangs ont évidemment des configurations très différentes, et cela n'est pas anodin. La forme, la profondeur, la qualité du fonds, l'origine et la quantité des eaux que reçoit un étang, l'exposition : de tout cela, va dépendre la façon dont il se vide (et le temps que l'on mettra pour parvenir à le vider), le taux d'évaporation, le type d'empoissonnement que l'on privilégiera. Tant de paramètres entrent en jeu que l'on s'entend généralement pour dire qu'il n'est pas un étang qui ressemble à un autre.

Différentes catégories d'étangs permettent de diversifier la production, et de suivre «l'élevage » à ses différentes étapes. Les petits étangs, d'à peine quelques hectares, ceux destinés à la ponte ou au développement des «feuilles», sont idéalement situés le plus haut en amont, de façon à ce que l'on puisse en contrôler le fond, et être sûr de pouvoir éviter la présence de prédateurs, perches ou brochets, puisque, tout en haut de la ligne, ils ne recevront que les eaux de pluie ou de ruissellement, et non l'eau d'autres étangs avec ses risques.

Tout cela est de la plus grande importance pour comprendre le monde des étangs : le contrôle du cycle de la pisciculture n'est pas un mince enjeu. 
Perdre le moins d'eau possible

Au Portugal, dans la région de Melgaço évoquée, pas d'organisation centralisée, ni de contrôle du groupe des ayants droits, mais des règles connues de tous, vivement revendiquées, perpétuées d'année en année durant la saison d'été qui inaugure la réglementation. En Brenne le coutumier a longtemps eu force de loi, et s'il ne l'a plus depuis à peine deux décennies, on s'y réfère toujours. Mais beaucoup de ceux qui ont acheté des étangs récemment l'ignorent: les propriétaires en Brenne n'ont pas tous une attache locale comme c'est le cas à Melgaço.

Car pour parvenir à pêcher l'ensemble des étangs, il existe bien une façon de faire, un ordre, précisément prescrit par les us et coutumes, un droit coutumier écrit qui s'est formulé et affiné avec le temps: il permet une organisation de la gestion de l'eau à une échelle large. Tous reliés entre eux par des fossés d'écoulement de l'eau ${ }^{10}$ et de ce fait fortement dépendants les uns des autres, les étangs doivent théoriquement — introduisons d'emblée une réserve — être vidés au fur et à mesure des pêches, d'aval en amont.

Le premier étang vidé en aval recevra les eaux de l'étang situé plus haut, et ainsi de suite d'étang en étang. L'eau se voit ainsi chaque fois récupérée de façon à ce que, du moins dans le système idéal et dont le fonctionnement serait relativement simple, il n'y ait que l'eau d'un seul étang qui soit perdue, celle de l'étang le plus en aval, pêché en premier, qui se jette dans la rivière. L'étang situé le plus en amont ne pourra, lui, être rempli que par les eaux de pluie et de ruissellement.

Et la gestion des eaux s'opère ainsi à différents niveaux. Les propriétaires des grands étangs qui jalonnent le cours d'un même ruisseau ont à se concerter et à coordonner leurs dates de pêche; ils sont d'ailleurs en relation, parfois se fréquentent, ou, si ce n'est pas le cas, parviennent éventuellement à s'entendre par l'intermédiaire de leurs négociants respectifs ou surtout de leurs gardes qui vivent sur place. Ces derniers eux-mêmes bien entendu se connaissent, peuvent aussi faire partie d'un même réseau d'amitié et d'entraide, ils sont en contact de toute façon, se préviennent, s'informent les uns les autres.

Mais chacun des grands étangs a également sa propre ligne d'étangs, dont les eaux vont à un moment ou à un autre venir se rassembler pour le remplir.

Cette technique de vidage des étangs, qui opère de proche en proche, est précisément décrite et recommandée dans la dernière version du code coutumier publié, celle de 1967, notamment en ses articles 88 et 89 :

Article 88. Lorsque des étangs se commandent, c'est-à-dire se vident l'un dans l'autre, le propriétaire de l'étang inférieur doit pêcher le premier son 
étang. S’il ne veut pas le pêcher, il doit au moins en baisser le niveau de manière à rendre possible la pêche de l'étang supérieur.

La deuxième phrase de ce même article laisse évidemment toute latitude à l'aval, nous sommes en régime de propriété privée, et il n'a jamais été question en ses propres terres de ne pas agir à sa guise. Libre au propriétaire donc de ne pas pêcher et ainsi de ne pas vider son étang, mais il ne doit pas pour autant oublier l'autre, il a obligation de ne pas entraver le droit de l'amont à pêcher, et devra baisser son niveau de façon à ce que l'eau puisse passer.

L'article suivant toutefois oppose une condition qui peut justement sembler paradoxale en ce qu'elle vient limiter la priorité de l'aval:

Article 89. Celui qui veut pêcher l'étang supérieur doit avertir un mois à l'avance le propriétaire ou le fermier de l'étang inférieur.

Aucun des termes utilisés aujourd'hui (chaîne, lignée, chapelet) et qui traduisent le lien ou la dépendance entre les étangs, n'est repris dans le coutumier, mais dans l'article 88, une phrase entière marquée par le verbe «se commander » indique, de façon on ne peut plus claire et assurée, leur interdépendance.

La juxtaposition des deux articles successifs alimente le débat en Brenne: de deux choses l'une en effet, ou il y a absolue priorité de l'aval telle qu'elle semblait annoncée, ou non. Aucune ambiguïté ne devrait être possible. S’il y a toujours priorité de l'aval, comment admettre qu'il suffise au propriétaire de l'amont de prévenir un mois à l'avance pour avoir le droit pour lui ?

Dans cette région où les étangs ne bénéficient que des eaux du ciel, l'ordre de vidage des différents étangs pour les pêches a bien été conçu en vue de perdre le moins d'eau possible. En cela, et par la communauté d'obligations et de devoirs (certes différents selon les périodes historiques) qu'il suppose, il relève d'une véritable gestion hydraulique d'ensemble. L'esprit s'en est transmis, il s'est affiné, précisé, non sans tâtonnements, et parfois, on le voit dans les articles 88 et 89, avec des ambiguïtés qui en font aussi l'extrême fragilité.

Perdre le moins d'eau possible, parvenir à ne jamais en gaspiller: la notion d'économie de l'eau est dans cette société une préoccupation constante, elle est essentielle à l'intelligence du système.

L'allusion à la période monastique, et à une perfection hydraulique qui lui serait associée, est récurrente dans le discours de certains grands propriétaires aujourd'hui. Sans doute parce que dans le contexte de désordres accrus, dus notamment à l'accroissement du nombre de propriétaires dans un territoire qui 
se morcelle, l'image mythifiée d'une harmonie originelle dans la conception d'ensemble du système d'étangs reprend force. Il est évident pour tous, en ce qui concerne les anciens étangs, que situation topographique de plans d'eau, pentes et écoulements possibles et équilibre entre arrivée d'eau et déversoirs permettant de résorber les surplus, ont été minutieusement pensés et calculés pour utiliser l'eau au mieux. Certaines conventions très anciennes établies entre étangs en sont à leurs yeux la preuve.

Les termes de ces conventions se voient parfois consignés dans les actes mêmes de propriété et réitérés à chaque transaction de biens. Il en est ainsi, et je ne prendrai que cet exemple, le plus remarquable, pour deux grands étangs prestigieux très proches l'un de l'autre, mais situés sur deux bassins versants différents, et dont l'un, le Gabriau, est très pauvre en eaux de ruissellement, et l'autre, la Gabrière, ${ }^{11}$ situé sur un large bassin versant, peut se remplir aisément. Pour pallier ce défaut, un droit de prise d'eau du Gabriau sur la Gabrière a été prévu par une bonde de surface située sur la Gabrière, afin de permettre un passage d'eau d'un étang à un autre, jusqu'à « recouvrir d'eau » le Gabriau, après qu'il ait été vidé pour la pêche, et au cas où il n'aurait pas assez d'égout pour se remplir. Une répartition des eaux plus équitable a ainsi été prévue et rétablie.

\section{Interdépendances}

Les deux opérations, dites «mise en tire » et «mise en pêche », sont essentielles au bon fonctionnement du système. Il faut, pour arriver à pêcher un étang, parvenir à le vider progressivement de la presque totalité de son eau de façon à ce que le jour de la pêche toujours fixé à l'avance avec les négociants, le ramassage des poissons à la filanche ${ }^{12}$ ou à l'épuisette au sein d'un espace restreint enserré par un filet, soit aisé. La mise en tire désigne cette opération de vidage qui doit s'effectuer en douceur pour ne pas malmener le poisson. Elle suppose une diversité de savoir-faire liée aux différences des étangs.

Les dates de début de mise en tire, où l'on commence à lever les bondes, et les temps de mise en tire diffèrent d'un étang à l'autre, surtout selon leur ampleur. Ce sont des éléments essentiels à prendre en compte pour arriver à ce qu'un étang soit prêt à être pêché le jour convenu. Mais un bon déroulement de l'opération suppose aussi une bonne volonté de l'amont qui doit retenir ses eaux.

Les documents d'archives nous laissent percevoir durant tout le xix ${ }^{\mathrm{e}}$ siècle, les réactions vives des hommes d'étangs à toute intervention de l'administration d'État dans leurs pratiques. ${ }^{13}$ Car l'administration, dans le noble projet d'« assainissement de la Brenne » qu'elle s'est fixé, est contrainte d'interdire à maintes reprises la levée des bondes des étangs pour pourvoir procéder au curage des 
ruisseaux alors encombrés de déchets végétaux. Ou bien encore elle ordonne de fermer les bondes d'un grand étang, car des plaintes lui sont parvenues d'émanations fétides et jugées dangereuses pour la santé. Chaque fois les hommes se rebiffent: ils veulent être totalement libres de déterminer à quelle date ils doivent commencer à vider leurs étangs pour les amener en pêche, et s'évertuent à expliquer à une administration souvent ignorante, au xix ${ }^{e}$ du moins, les impératifs de respecter les dates de mise en tire pour arriver à pêcher à la date juste et ainsi ne pas troubler l'ordre de vidage, ne pas risquer de perdre l'eau: là est bien l'enjeu.

L'équilibre entre propriété privée et réglementation publique reste toujours délicat de nos jours. Et cette extrême sensibilité des propriétaires exploitants d'étangs à toute intervention administrative dans les décisions concernant leurs étangs, et particulièrement les dates de vidage, est la même aujourd'hui. Elle a eu l'occasion de s'exprimer avec force lors de la parution de la loi sur l'eau de 1992, où il était question, entre autres, de réglementation du vidage des étangs. Les énoncés sur les demandes préalables d'autorisation de vidage, qui semblaient devenir obligatoires, avaient provoqué de vives réactions du Syndicat des exploitants d'étangs. Il y voyait une immixtion intolérable dans la gestion des étangs.

«Mettre en pêche » un étang consiste à le vider suffisamment la veille même de la pêche pour rendre possible le travail des pêcheurs l'aube venue. Si l'opération se passe bien, à l'aube, l'étang est presque entièrement vide d'eau, sauf en sa partie plus profonde, «la pêcherie », où le poisson sera puisé. Mais durant toute la journée encore, les hommes restent vigilants, inquiets, surveillent le ciel, se méfient toujours d'une manœuvre de l'amont: que la pêche se passe au mieux dépend aussi toujours, et jusque pendant le déroulement de la pêche, de ceux du dessus. Qu'un propriétaire ou un garde d'étang juste en amont vide sans prévenir, et sans se poser la question de savoir ce qui se passe à l'aval, et le déroulement de la pêche est menacé, «Trois heures de pluie et vous ne pouvez pas pêcher, alors si on vous vide un étang de 3 hectares...».

Le jour de la pêche, la pêcherie, qui ne fait pas plus que quelques ares près de la bonde, forme comme un cercle d'eau où tout le poisson se voit concentré. Une courante d'eau est alors nécessaire, le plus souvent pendant la pêche même, afin d'oxygéner le poisson réuni dans une aussi faible quantité d'eau et en souffrance, pour peu que le temps en tout début de saison soit encore trop chaud, ou que la pêche s'avère plus longue que prévu ; ou encore juste après la pêche, pour sauver le fonds de poisson resté dans l'étang qui ne se remplira que progressivement. Sur les grands étangs, le seul égout des vases découvertes, l'égout naturel, peut suffire, mais pas toujours, et sur les petits étangs une arrivée d'eau 
est indispensable. Une petite pluie en fin de pêche peut aussi être très utile, elle est toujours la bienvenue, l'étang demeure cependant tributaire de l'amont. Et l'on doit s'assurer déjà la veille de la pêche, en dehors des pluies imprévisibles, que l'on ne manquera pas d'une arrivée d'eau.

Si le propriétaire qui pêche possède également l'étang en amont, l'opération est évidemment plus simple, il peut avoir commencé à vider cet étang avant de pêcher celui de l'aval, il est ainsi certain de disposer d'eau pendant la pêche, d'avoir un filet d'eau qu'il peut régler lui-même, mais un propriétaire le dit en riant : « On ne peut pas avoir toute la Brenne à soi !» Alors le plus souvent, on doit demander au propriétaire de l'étang de l'amont de lever un peu sa bonde avant la pêche de l'aval. Outre la démarche, sur laquelle nous reviendrons, cela n'est pas si aisé, car il faut connaître les temps d'écoulement de l'eau d'un étang à l'autre - ce sont là des données capitales -, qu'il s'agisse exceptionnellement pour une raison imprévue d'envoyer un gros coup d'eau, ou que l'écoulement se fasse en douceur pour un apport d'eau nécessaire au moment de la pêche.

Ainsi la maîtrise de l'eau, dans ce territoire où l'on ne dispose pas d'eau de sources mais seulement des eaux de pluie, ce serait de posséder l'étang du dessus pour disposer d'une courante d'eau lors de la pêche. Mais la maîtrise de l'eau s'avère tout aussi importante pour pratiquer véritablement le cycle de la pisciculture, car elle permet de contrôler le fonds de l'étang supérieur et d'être sûr que des espèces indésirables ou des prédateurs ne s'y trouvent pas et ne passent plus bas. La vraie maîtrise de l'eau que tout propriétaire rêve d'avoir serait de pouvoir disposer d'une « chaîne » d'étangs qui se suivent depuis le tout premier en amont et de pouvoir mettre en œuvre le cycle de la pisciculture dans son ensemble, sans avoir affaire aux autres: c'est ainsi qu'il faut comprendre les stratégies foncières des grandes familles installées dans les terroirs qui, durant tout le $\mathrm{xix}^{\mathrm{e}}$ siècle, pratiquent une politique d'échanges de biens. Ne pas avoir d'enclaves dans ses terres, être maître chez soi aussi bien pour la chasse que pour l'exploitation d'étangs, tous les propriétaires exploitants d'étangs y aspirent, certains d'entre eux en ont connu un temps le privilège.

Si l'on y parvient, la maîtrise de l'eau est essentielle. Assurer sa propre production est associé chez les grands propriétaires à un privilège plus large : «Autrefois, on avait tout, le filet, le matériel, les caisses, la main-d'œuvre, les domestiques. » Une sorte de fierté était attachée à cette priorité qui marquait aux yeux de tous qu'on était maître chez soi, que l'on avait la parfaite maîtrise du cycle piscicole, que l'on ne dépendait pas des autres et encore moins de négociants pour se procurer de l'alevin.

Pouvoir être maître de l'eau chez soi en Brenne est aussi ce qui pousse certains 
propriétaires aujourd'hui à vouloir créer sur leurs terres de petits étangs qui se suivent, ou qui viennent s'intercaler entre d'autres qui leur appartiennent; ou encore un propriétaire d'étangs et de bois, à louer étangs et bois qui jouxtent ses terres, de façon à inclure des étangs dans sa propriété et lui permettre, par le contrôle d'un certain nombre d'étangs, de diversifier sa production, en même temps que d'avoir un domaine de chasse suffisamment vaste.

Aux conventions entre étangs, notifiées par écrit dans les actes de vente des étangs, comme celle dont il a été question et qui lie intimement les deux étangs Gabriau et Gabrière, s'ajoutent les arrangements qui, eux, ont une place décisive dans le fonctionnement du système. Ils peuvent être de toutes sortes.

On a souligné l'importance de pouvoir disposer d'une courante d'eau venue de l'amont pour l'étang que l'on pêche, et qui permet d'oxygéner le poisson réuni dans l'espace restreint de quelques ares d'eau, ou après la pêche pour sauver le poisson que l'on a laissé en empoissonnement. En amont, lever les bondes pour permettre une petite arrivée d'eau en aval: cette faveur n'est jamais refusée, elle relève d'une solidarité dans le monde des étangs: «En cas de pépin, en cas de besoin, il n'y a pas de propriétaire qui refuse d'ouvrir plus haut et de laisser couler l'eau. » Elle s'accompagne, dans le discours, de l'énoncé maintes fois réitéré d'un principe éthique fondamental dans cette société: «On ne refuse pas l'eau », principe dont on verra ultérieurement qu'il peut avoir une large acception, qu' il joue en différents espaces et différents lieux de la gestion de l'eau, et en cela contribue fortement à la cohésion de la société locale.

Toujours au moment de pêcher, le propriétaire de l'étang en aval, cette fois, peut demander à ceux de l'amont de baisser un peu le niveau, de « faire une marge », une marge de sécurité afin d'éviter que leurs étangs ne débordent le jour de la pêche de l'étang situé en aval. Cela était encore possible sur certaines lignes d'étangs lorsque les propriétaires étaient moins nombreux. Mais aujourd'hui, on s'adresse au propriétaire le plus proche en amont, on lui demande de retenir quelque peu les eaux de son étang qui reçoit toutes celles encore au-dessus, pour que l'on parvienne à pêcher sans surprise.

Il paraît inadmissible, inimaginable qu'on ne permette pas à l'autre de vider son étang, en somme qu'on ne respecte pas les usages, au point que dans le discours, les usages prennent force de loi.

Ces types d'entente très précieux — retenir l'eau pour permettre à l'aval à pêcher, envoyer, «donner » de l'eau le jour de la pêche — se transmet de génération en génération parmi les grandes familles vivant en Brenne, ou entre ces dernières et les familles d'agriculteurs auxquelles elles sont liées, et de part et d'autre, on y tient doublement: pour des raisons techniques à l'évidence 
capitales et par tradition. «Déjà du temps de mon grand-père, prévenir celui de dessous qu'on lève l'étang, lui donner un peu d'eau quand il a pêché son étang, c'était l'habitude, on continue... », «Son père était là avant lui, mon père était là avant moi, on se connaît et on fait ce qu'ont fait nos pères ». Ainsi se dit la transmission d'usages que l'on respecte par égard aussi pour une tradition familiale.

Même si l'idéal reste toujours de ne pas avoir affaire aux autres, de posséder soi-même plusieurs étangs successifs.

Décalages entre ordre coutumier et pratiques

Lorsque les hommes parlent de l'ordre du vidage des étangs d'aval en amont pour la pêche sur une ligne d'étangs, ils utilisent un vocabulaire qui relativise d'emblée l'ordre coutumier et place les choses sur le terrain bien concret des aléas toujours possibles, des accommodements et arrangements divers ou, au contraire, de manœuvres individuelles conduisant au plus grand désordre dans la gestion des eaux : «En principe, on devrait d'abord pêcher... », « La logique voudrait que... mais... », «Théoriquement, on devrait... ». Les mêmes termes sont employés pour évoquer l'obligation, qu'impose à l'amont l'article 89 du droit coutumier, d'avertir un mois à l'avance le propriétaire ou le fermier de l'étang inférieur. Longtemps, et du fait évident de difficultés, cet article a dû être pris par certains avec le plus grand sérieux, au point que l'usage était plus précisément d'envoyer une lettre recommandée au propriétaire de l'aval pour l'avertir que l'on allait pêcher et qu'il devrait se préparer à recevoir, le jour fixé, l'eau de l'étang. Dans la pratique, la lettre recommandée avait dans les esprits fini par relever du coutumier lui-même qui n'indique pourtant pas de procédé précis pour prévenir l'aval. L'arrangement intervient ou non dès le début, «On s'arrange, c'est une entente tacite », on se rencontre, ou l'on se prévient par téléphone, on en parle, ou les gardes des étangs se préviennent. Mais, avec ceux qui ont un caractère peu accommodant et font des histoires, la lettre recommandée est un moyen qui s'est, ici et là, progressivement imposé.

En principe donc, selon l'article 88 du droit coutumier, et en toute logique si l'on veut perdre le moins d'eau possible, c'est l'étang le plus en aval qui doit être pêché le premier. Mais des dérogations ont lieu, cela arrive par commodité et pour toutes sortes de raisons dont on peut convenir entre soi. Lorsque sont évoquées ces situations très précises, on se démarque avant toute chose de ceux qui ne respectent pas l'ordre coutumier, le méprisent ou l'ignorent. Il ne s'agit que d'essayer, ponctuellement, entre voisins, par courtoisie, de s'adapter, une année d'accepter les craintes ou les souhaits de l'autre, ou de trouver la solution qui s'avère la meilleure pour tous sur le terrain avec les étangs que l'on connaît, 
de déroger à la règle tout en essayant de préserver le plus d'eau possible dans le système: on est là dans le domaine concret des pratiques.

Ainsi, tel propriétaire a empoissonné cette année en nourrains ${ }^{14}$ un petit étang qu'il aimerait pêcher tardivement du fait que l'étang, proche de chez lui, peut être surveillé de la prédation des cormorans, et il préfère attendre un peu plus qu'à l'accoutumée et pêcher après l'amont; tel autre aimerait qu'une partie de sa famille puisse être là au moment de la pêche, or cette année exceptionnellement, elle ne pourra venir en Brenne qu'à telle date, ce qui amènerait à pêcher plus tard qu'à l'accoutumée... Tout se passe à l'amiable, «d'un commun accord », on trouve la solution.

Le système est évidemment en constant mouvement, et des réajustements sont toujours nécessaires. Du fait d'héritages ou de ventes, des étangs changent de mains, de nouveaux étangs se créent, de nouvelles relations entre propriétaires doivent être établies, d'autres arrangements trouvés sur des fondements différents, les exigences bien sûr pouvant ne plus être les mêmes et le nombre des étangs concernés évoluer. Aussi, les accords, comme les moments de tension et les conflits, ne durent-ils qu'un temps, mais ils se renouvellent, se transforment éventuellement: ensemble et dans leur diversité, ils restituent l'esprit du système.

Sur certains étangs les propriétaires font tout pour que les propriétaires des terres en amont, souvent des agriculteurs qui ont créé des étangs sur leurs terres, respectent l'ordre du vidage des étangs, et pêchent après eux. Car l'eau accaparée, et retenue par les nouveaux étangs en amont, ne doit pas être perdue pour ceux de l'aval qui, avant la création d'étangs, la recevaient sous une autre forme. Les propriétaires d'étangs comptabilisent en effet les hectares d'eau retenus en amont évaluent la différence de qualité d'une eau qui autrefois leur arrivait de façon continue par ruissellement après les pluies. Mais ils vont plus loin dans le raisonnement et leur comptabilité: cette eau retenue ne correspond pas à la totalité de l'eau qu'ils auraient reçue si les nouveaux étangs en amont n'avaient pas été créés. L'eau qui, dans le meilleur des cas, leur sera finalement envoyée après avoir été longtemps retenue, se sera en partie déjà évaporée et sera un peu moins importante que l'eau de ruissellement. Il y a bien ainsi déperdition. Du côté des agriculteurs en amont, propriétaires d'étangs du même bassin versant, cela paraît excessif, on est un peu interloqués par un discours aussi argumenté et une telle énergie mise en ouvre pour contrôler l'eau que les propriétaires traditionnels en viennent selon eux à « sacraliser». Il est vrai que dans les anciennes familles, l'ordre coutumier visant à préserver l'eau est tellement intériorisé que l'on accepte difficilement que certains y dérogent. 
Ailleurs, il faut chaque année «jongler » pour ne pas perdre d'eau. «Jongler», le mot revient fréquemment lorsque l'on parle de l'organisation du vidage pour les pêches, jongler avec les dates, jongler pour récupérer le plus d'eau possible, jongler avec les aléas de toutes sortes comme l'arrivée de pluies ou un gel soudain qui paralyse les pêches. Sur une autre ligne d'étangs où les étangs sont en nombre, de l'avis de tous, «si on ne s'entend pas, c'est invivable ». Et l'on n'imagine pas que l'on puisse ne pas être solidaires.

Mais même sur un bassin versant où le ruissellement est largement suffisant, où l'on ne risque pas de manquer d'eau, si en amont un propriétaire propose une année de pêcher avant l'aval, alors en aval on ne peut s'empêcher de penser qu'il s'agit là d'une eau malheureusement « perdue ».

«Sauvage » : c'est ainsi qu'est significativement désigné le vidage intempestif d'étangs auquel on procède sans que l'on prévienne son voisin de l'aval, vidage qui, dès lors, peut avoir lieu aussi bien pendant que l'aval est en pêche. Sauvage en ce qu'il ne respecte pas les autres, en ce qu'il ignore ou méprise l'ordre coutumier élaboré par les hommes. Le terme est fréquemment repris dans les mémoires ou les rapports officiels qui existent sur le sujet mais aussi en Brenne lorsque est évoqué le vidage par de nouveaux propriétaires. De même les écrits se font-ils écho de la création anarchique d'étangs. «Sauvage », « anarchique » : les termes sont du même ordre qui renvoient de façon éloquente à des comportements hors normes qui ne sauraient être supportés par la société des étangs et auxquels pourtant, pour parvenir à survivre, elle doit s'adapter.

L'image d'une perfection hydraulique ancienne et perdue, qui reste une référence pour les grands propriétaires, les hommes dans la Dombes, autre région d'étangs, semblent la partager. Eux aussi, ils l'évoquent avec nostalgie, déplorant que, avec le morcellement foncier, le démantèlement des chaînes et l'apparition de tous ces nouveaux propriétaires qui ne respectent pas les usages, le système d'eau soit « cassé $»^{15}$. Ce dernier terme est en lui-même éloquent quant à la perception que l'on se fait d'un système comme d'un mécanisme sans faille, parfait, immuable comme s'il n'était pas toujours soumis à des ruptures possibles.

En zone humide, l'eau précieuse

Reprenons ce qui vient d'être rapidement décrit: l'ordre strict que recommande le droit coutumier pour parvenir à récupérer au mieux l'eau d'étang à étang, et lorsque l'ordre est quelque peu bousculé, les tentatives d'arrangements dans lesquelles on s'épuise parfois pour ne pas perdre d'eau, cette attention aiguë portée à la pluviométrie, les calculs, auxquels se livrent certains exploitants d'étangs, des déperditions d'eau causées par la création d'étangs en amont qui 
détournent les eaux de ruissellement, et dès lors la prise en compte précise des pertes par évaporation l'été, les efforts déployés pour récupérer malgré tout le plus d'eau possible, "en tête de chaîne », pêcher de bonne heure, le plus tôt possible pour pouvoir bénéficier des pluies de printemps... Ce sont autant d'éléments qui démontrent, aussi paradoxal que cela puisse paraître en zone humide, le caractère précieux de l'eau, l'importance qu'on lui accorde, l'attention extrême qu'on lui porte. Il est toujours question de ne pas gaspiller l'eau ou du moins d'en gaspiller le moins possible, surtout de ne pas la perdre, d'où toutes les manœuvres lorsque l'ordre d'aval en amont ne peut être respecté, pour l'« emmagasiner », la «stocker », la garder en attente avant de la lâcher afin de remplir ultérieurement les étangs : «jongler », c'est cela.

Lorsqu'on s'insurge contre l'inconscience de ceux de l'amont qui agissent sans prévenir et ne se préoccupent pas des conséquences pour l'aval, qu'une manœuvre incontrôlable a eu lieu et que l'eau est perdue, c'est bien la valeur de l'eau qui est mise en avant : «Il s'agit de ne pas perdre l'eau qui est notre richesse tout de même! »

Parfois, pour que tous parviennent à pêcher sur une ligne, être contraints de laisser passer dans son étang l'eau de l'amont, la laisser se perdre ensuite dans le ruisseau, sans qu'elle ait pu être récupérée par l'aval qui n'a pas encore pêché, cela fait véritablement mal au cœur à ceux qui s'y résignent et l'admettent très difficilement. Parmi les membres des anciennes familles, le caractère précieux de l'eau est si fortement intégré qu'ils éprouvent une vraie douleur à gâcher l'eau, pas seulement pour leurs propres étangs mais pour ne pas nuire à l'économie d'ensemble. Même sentiment exprimé avec force dans une autre société à étangs comme la Dombes où, pour un propriétaire, «perdre de l'eau équivaut à lui arracher le cœur $»^{16}$.

Il arrive que, par un souci d'éthique déclaré, l'on cherche à tout prix à s'adapter aux souhaits de son voisin: "Je ne pêcherai jamais sans m'entendre avec l'aval, je ne pêcherai jamais s'il ne récupère pas mon eau. » Il arrive aussi que de nouveaux propriétaires agriculteurs par exemple, qui se mettent à faire de la pisciculture, au début se révoltent contre tel grand propriétaire qui veut faire la loi sur la ligne d'étangs, mais ressentent ensuite le même sentiment de perte et de gâchis si eux-mêmes ont des problèmes avec l'amont, et adoptent vite alors le même discours... «Une averse et il ouvre son étang, vous vous rendez compte ! Ça, c'est tout de l'eau gâchée ! Bonne pour la rivière celle-là !»

Le « détournement» de l'eau par des étangs créés en amont peut être vécu comme une véritable usurpation, sentiment qu'il faut surmonter toutefois, car la loi ne l'entend pas ainsi: l'eau recueillie sur les terres en amont relève bien 
du propriétaire du fonds. Le sentiment est d'autant plus amer si les nouveaux propriétaires ne partagent pas les mêmes valeurs. Que l'eau dans cette société soit « sacrée », et qu'elle soit la richesse commune, les membres des anciennes familles aimeraient au moins que cela soit reconnu par tous.

Une richesse commune : si dans le discours on emploie le possessif lorsqu'il s'agit de l'eau, «Je lui envoie mon eau », «Je récupère son eau », «Je ne pêcherai jamais s'il ne récupère pas mon eau», ou encore «Je lui donne de l'eau» comme si l'eau était à soi, il est clair pour chacun que l'eau n'est propriété de personne mais bien de tous. L'eau ne fait que passer dans son étang, et si, à un moment précis, on la fait sienne au point de dire qu'on la donne, on sait que ce n'est jamais que pour un moment.

L'eau pour se parler

Rien de ce qui est évoqué et regretté — la faveur d'accorder de l'eau, les arrangements, les rapports courtois et respectueux entre les hommes — n'était strictement honoré par les hommes auparavant, pas plus que n'étaient strictement respectés les usages ancestraux, l'ordre dans le vidage. Sauf peut-être pour ce dernier point, il y a des siècles, lorsque tous les biens étaient dans les mains d'un même seigneur, ou aux temps monastiques lorsqu'un véritable système d'étangs se mettait en place sous le contrôle d'un seul, mais alors cela n'avait évidemment pas le même sens, ni la même valeur.

Ici et là, un ordre ancien auquel est associée l'harmonie dans la gestion des eaux est illusoire, les tensions et le désordre qui se sont incontestablement accentués aujourd'hui font partie de l'ordre même des choses. Mais l'image d'un ordre ancien, de l'entente si ce n'est de la perfection, de la cohésion tranquille du système, est forte, et se transmet. Elle n'est jamais que l'image que s'en font les hommes à un moment donné, une représentation des choses, rien de plus, qui ne résiste pas longtemps à l'analyse du fonctionnement réel du système.

Les attitudes égoïstes, les comportements individualistes, les caractères imprévisibles pourraient bien ne pas être exclusivement le fait de propriétaires des nouveaux étangs. Il en était sans doute de même entre les anciens propriétaires issus de grandes familles locales. Car cette notion d'ordre idéal, à laquelle les hommes se réfèrent si souvent, a toujours été indissociable du désordre possible, dont les divers coutumiers d'ailleurs et jusqu'aux plus anciens se font l'écho à travers le temps.

Un texte du début $\mathrm{du} \mathrm{xix}^{\mathrm{e}}$ siècle fait ainsi très explicitement allusion à «l'incurie ou aux mauvais procédés des usagers »: 
Le premier pêché sur chaque ligne - ordinairement le plus bas se remplit lorsque celui placé immédiatement au-dessus est mis en pêche. Sans cette précaution, quelquefois négligée par suite d'incurie ou de mauvais procédés des usagers, le fond d'une partie des étangs ne se couvrirait qu'imparfaitement dans les années sèches.

L'incurie pourrait correspondre à l'insouciance, à la non considération des autres, à l'individualisme, qui se sont assurément affirmés ces toutes dernières décennies. Tandis que «les mauvais procédés », ainsi désignés par un homme réservé et courtois de l'époque, pourraient renvoyer, plus qu'à une méconnaissance des usages, déjà à un refus de les reconnaître ou, pire encore peut-être, à quelque manœuvre dans le but de nuire à l'autre. Rien que de très actuel.

Difficiles relations de voisinage, vieilles inimitiés, rancunes ou jalousies du moment se répercutent dans la gestion même des étangs, y trouvent le moyen efficace de s'exprimer, l'occasion renouvelée de se dire. Et l'on mesure mieux alors, en dehors des prescriptions du coutumier, l'importance essentielle des arrangements, car il y a bien dans la gestion des eaux une possibilité de réplique immédiate, de brimade bien concrète, de pression, de revanche, cela fait partie de l'ordre même des choses, au point qu'il n'est pas de conflit dont les traces restent durables.

Et à suivre les étangs et ce qui se passe sur chacun d'eux, mais aussi sur la totalité du réseau, de proche en proche, tout s'éclaire. Silencieusement, à travers l'eau, les faits prennent sens. Les répercussions des conflits, ayant trait à l'eau ou pas, les ressentiments suscités se lisent dans la gestion même de l'eau.

Mais cela va plus loin encore: les arrangements, eux-mêmes présentés comme le fait d'une société solidaire et qui agit au mieux pour la meilleure gestion des eaux, apparaissent intrinsèquement liés aux représailles possibles s'ils sont refusés... Les propriétaires de l'amont envoient bien une petite courante d'eau le jour de la pêche de l'étang en aval, alors que le propriétaire en aval par ailleurs se plaint sans cesse des agissements de l'amont... mais sur le service rendu, le propriétaire de l'aval, lui, a les idées claires: « ils y sont bien obligés, c'est normal ! Et s'ils ne l'avaient pas fait, je peux après très bien refuser que l'eau passe! »

Ainsi, en cette terre de propriété privée, on est tenu les uns aux autres. Si « on ne refuse pas l'eau », beau principe énoncé avec conviction et noblesse et qui fait l'unanimité, c'est aussi qu'on ne peut prendre le risque de la refuser. La tension est au fondement de l'équilibre auquel on parvient et qui s'instaure du fait même des pressions entre amont et aval: le coutumier, dans le paradoxe 
apparent des deux articles qui se suivent, n'exprime rien d'autre.

Comme dans toute société où elle est une ressource précieuse, parce qu'elle l'est, l'eau se révèle être aussi, dans cette société à étangs solidaires, un langage privilégié. Par l'eau on sait pouvoir faire entendre sa voix, régler ses comptes: d'amont en aval, d'aval en amont, les hommes sont étroitement liés par l'eau. Efficace, le langage de l'eau est aussi le mieux entendu, et il atteint toujours son but.

\section{L'eau des étangs précieuse pour les agriculteurs}

Droit ou tolérance

Le caractère précieux de l'eau apparaît de façon plus immédiate, lorsqu'on quitte l'exploitation piscicole des étangs pour aborder les alentours, le monde rural. Car l'eau des étangs est d'une importance capitale dans l'univers paysan, pour la possibilité qu'elle offre d'y laisser pacager et s'abreuver le bétail en saison chaude, et de faucher les joncs nécessaires aux litières des étables ou aux toits des habitations. ${ }^{17}$ Cet usage plus particulier, dans une région où les céréales n'étaient pas assez abondantes et où dès lors manquait la paille, était loin d'être secondaire. Aujourd'hui, on se souvient encore de l'époque pas si lointaine, quelques décennies seulement, où les herbes aquatiques — joncs, rauches et roseaux étaient toujours recherchées pour les litières.

Pacage, abreuvage et fauchage peuvent faire l'objet de droits écrits, parfois de conventions temporaires, qui valorisent de façon significative les terres autour des étangs. Mais ils relèvent aussi, et le plus souvent, de simples tolérances de la part des propriétaires de l'eau, si essentielles toutefois pour les agriculteurs, que ces derniers ont tendance à les considérer comme des droits. De même, les servitudes qui grèvent les étangs et qui ne devraient être que saisonnières, sont-elles souvent considérées comme permanentes par ceux qui font de l'élevage. De là, et cela est une constante, les protestations des propriétaires d'étangs contre ce qu'ils jugent des pratiques abusives.

L'ambiguité plane toujours localement dans les esprits entre droits et simples tolérances, mais toute une palette subtile d'arrangements tacites a été possible parce que les hommes qui travaillent sur les terres en bordure des étangs ont à subir les inconvénients de cette proximité: les crues soudaines, l'inondation des prés, et, pire, lorsque la terre était encore travaillée, l'inondation des champs avec la perte sûre d'une partie des cultures. Aussi la tolérance, au moment de la saison d'été ou de périodes sévères de sécheresse, de laisser pacager les bêtes sur l'étang et de leur permettre de s'abreuver, est-elle vécue du côté des propriétaires comme une compensation légitime pour les dommages causés par les eaux, 
«une sorte de monnaie d'échange » comme l'avancent certains.

Les contraintes qu'imposent les périodes de crue et de sécheresse dans leur alternance, et la question toujours litigieuse des limites entre eaux et terres, lient ainsi étroitement les hommes de la terre et les hommes d'étangs. Elles sont au fondement du système social en Brenne. La nécessité vitale de l'eau des étangs pour l'élevage - et longtemps pour la survie même des hommes -, que les décisions politiques d'assécher les étangs à l'époque révolutionnaire, puis encore au xix ${ }^{\mathrm{e}}$ siècle, avaient révélée de façon brutale, scelle jusqu'à nos jours la dépendance plus large des agriculteurs par rapport au monde des étangs.

Cette latitude précieuse pour le riverain de laisser s'abreuver et pacager ses bêtes autour d'un étang, lorsqu'elle fait l'objet d'un droit, est toujours mentionnée dans les actes notariés lors des transactions diverses qui concernent l'étang frappé de servitudes ou les terres qui bénéficient du droit. Le propriétaire de l'étang se voit expressément interdit d'assécher l'étang, et lorsqu'il choisira de le laisser en chômage, l'étang ne devra pas le rester plus d'une année pour ne pas nuire au bénéficiaire; bien entendu, le droit sera suspendu pendant l'année où l'étang est en assec et éventuellement cultivé.

La différence avec la Dombes, qui connaît une alternance régulière sur ses étangs entre exploitation de la terre et évolage, est flagrante. On ne peut en Brenne mettre à sec fréquemment un étang et en enlever l'eau à ceux qui en ont un besoin vital durant la saison d'été, ni suspendre à intervalles rapprochés les possibilités d'abreuvage, qui sont depuis toujours la condition du maintien de l'élevage.

Le plus souvent l'abreuvage relève d'une tolérance du propriétaire de l'étang. Mais alors tous les arrangements tacites existent, eux-mêmes constamment susceptibles de nouvelles négociations et d'aménagements selon l'évolution de la relation entre propriétaires d'étangs et agriculteurs riverains ou proches. Ainsi dans un contexte tout récent, un exploitant agricole a-t-il demandé au propriétaire d'un petit étang de le laisser mettre ses bêtes en pacage sur les rives. Le propriétaire lui a accordé cette faveur, mais à la condition, et la transaction prend alors toute sa valeur pour le propriétaire, de pouvoir hausser le niveau ordinaire de l'étang de 15 centimètres. L'agriculteur accepte, car pour lui l'avantage est aussi important, mais il n'est pas dupe et, très vite, il évalue la superficie de prairie gagnée par l'eau avec l'exhaussement du niveau. ${ }^{18}$

Autrefois, jusqu'à il y a quelques décennies, lorsque les hommes étaient disponibles dans les domaines et suffisamment nombreux pour que l'un d'eux aille garder les bêtes sur les étangs, pacage et abreuvage étaient des pratiques différentes. Alors, les hommes se souviennent, « les vaches naviguaient dans les étangs ». Mais comme partout, avec la raréfaction de la main-d'œuvre et 
la disparition des bergers ou vachers, les hommes ont dû installer des clôtures et laisser les bêtes seules dans les prés, sauf qu'ici, les délimitations se font dans l'eau de l'étang aux formes irrégulières que la clôture vient trancher, et les propriétaires sont vigilants. Rectiligne, une clôture risquerait de marque les limites de propriété, aussi, rappeler aux agriculteurs qu'abreuvage et pacage sont le fruit d'une tolérance et non pas d'un droit, ou, plus justement, car ainsi on ménage les riverains, le leur faire sentir (et chacun le fait à sa façon), est un souci partagé des propriétaires. Et il est vrai que nombre d'exploitants agricoles, lorsqu'ils sont fermiers, ne se préoccupent guère de savoir si l'abreuvage des bêtes est un droit ; à leurs yeux il l'est, alors qu'importe si, lors de la lecture des baux, on ne trouve pas trace d'une phrase sur les droits éventuels; la question n'est pas à discuter. Ainsi aux droits et arrangements possibles, s'ajoutent les pratiques sans concertation aucune.

Compensation légitime pour les dégâts causés par les débordements d'étangs sans doute, mais cette tolérance à son tour devient une faveur dont le propriétaire attend d'autres avantages: la surveillance de l'étang, un coup de main éventuel, surtout la participation active et toujours bénévole aux pêches d'étangs qui exigent une mobilisation de main-d'œuvre. Participation longtemps vécue comme un privilège, reçu ou accordé. Le terme d' «invitation » par le propriétaire est d'ailleurs en usage. Des clauses particulières existent aussi pour certains étangs, explicites quant aux services qui peuvent être rendus lors des pêches. On le voit, la relation entre propriétaires d'étangs et agriculteurs alentour ne se réduit pas au lien créé du fait des usages d'abreuvage et de pacage, elle s'élargit encore au-delà. Droit ou tolérance de laisser les bêtes sur l'étang supposent encore une grande diversité d'échanges.

La présence du bétail sur les étangs a aussi en retour des avantages pour l'eau d'étang qui s'en trouve fertilisée, et dès lors pour la pisciculture. Les propriétaires exploitants ne l'ignorent pas, ils en tirent profit, et les agriculteurs sont conscients que la tolérance qui leur est accordée n'est pas elle-même sans contrepartie. Bénéfiques pour le poisson, les effets du pacage le sont également pour le gibier d'eau comme la bécassine et les petits échassiers. Les propriétaires profitent aussi du pacage des bêtes pour la chasse sur les étangs, et ils y trouvaient encore un autre avantage, avant que le faucardage ou les ragondins ne suppriment la végétation : les bêtes empêchaient les pousses de se développer et libéraient une part de surface d'eau.

Mais avec l'apparition d'une nouvelle catégorie de propriétaires, amateurs de chasse au gibier d'eau, et les transformations des pratiques d'élevage, les choses ont changé. Le troupeau des bêtes qui paissent est aujourd'hui beaucoup plus 
important, comme les agriculteurs éleveurs le reconnaissent. Non seulement le prélèvement en eau est loin d'être négligeable, ${ }^{19}$ mais les vaches qui ne sont plus surveillées peuvent monter sur les chaussées, les endommager, abîmer les bordures, par leur piétinement trop important, et y former des trous; si la terre est argileuse, un troupeau de soixante bêtes défonce le sol, les vaches s'y empêtrent jusqu'au ventre.

Le développement de la chasse, qui ouvre les étangs à une fréquentation nouvelle, est aussi vite devenu une source de conflit entre agriculteurs et chasseurs. Ces derniers se plaignent auprès des propriétaires qui leur louent des étangs, que le bétail peut survenir brusquement dans l'étang, effrayer et chasser le canard, et de façon générale de ce qu'il dérange le gibier d'eau. Les propriétaires aménagent tant bien que mal la cohabitation.

Parfois d'ailleurs, à l'occasion d'un changement de propriétaire ou d'une succession, les droits peuvent être transformés, le droit de pacage tombe, on ne conserve que l'abreuvage, et l'abreuvage lui-même s'aménage. Le « couloir d'abreuvage » est encore une étape dans l'évolution des accords à propos de l'utilisation des eaux d'étangs. Il restreint l'accès à l'étang, évite la dispersion des bêtes dans les eaux. Les agriculteurs, eux, en mesurent déjà les désavantages, nombreux.

Le lien entre les inconvénients dus aux débordements des eaux d'étangs, les dommages qui en résultent et la tolérance sur les étangs de la fréquentation des bêtes, propriétaires d'étangs et agriculteurs éleveurs l'ont clairement à l'esprit. Si ces derniers ont l'art, sans entrer dans le détail, du raccourci évocateur, «L'hiver, on a un mètre de flotte, l'été, nos bêtes boivent dans ces étangs », les uns et les autres connaissent parfaitement les différentes causes possibles des crues. Et les propriétaires, qui évoquent la tolérance de pacage et d'abreuvage comme « compensation », voire «monnaie d'échange » pour les dommages causés par les eaux, ne sont pas vraiment sereins, car ils savent aussi leur part de responsabilité dans certaines inondations. Soit qu'ils n'aient pas entretenu les fossés en aval de l'étang, soit que les déversoirs des étangs ne suffisent pas à évacuer l'eau en cas de fortes pluies, ou que les grilles au-dessus des déversoirs ne soient pas régulièrement libérées de ce qui entrave le passage de l'eau, soit que les bondes ne soient pas suffisamment importantes, soit, plus grave encore, que le propriétaire d'un étang, à l'occasion de travaux sur les ouvrages, ait fait remonter le niveau de l'étang en rehaussant la grande pierre plate qui se trouve à la base du déversoir...

Les choses ont changé plus largement. L'abandon des terres cultivées au profit des prairies rend le problème des débordements moins grave, les agriculteurs 
eux-mêmes en conviennent et en parlent spontanément: une prairie inondée, même si la crue dure plusieurs jours, ce n'est pas comme une parcelle de blé sous l'eau, et l'on est plus tolérant. L'amélioration relativement récente des ouvrages, l'invention notamment des bondes à déversoir intégré par lesquelles s'effectuent à la fois le vidage et l'évacuation du trop-plein, ont résolu en grande partie l'éventualité d'inondations. Les reproches constants faits au propriétaire ou au garde, de lever les bondes trop brutalement ou de ne pas surveiller les déversoirs, n'ont plus lieu d'être.

\section{Limites entre eaux et terres}

Lors même que l'abreuvage et le pacage relèvent d'un véritable droit, ce dernier a ses limites que les bénéficiaires ont tendance à oublier et à outrepasser. Il peut lui-même faire l'objet d'abus, aussi doit-il être surveillé par le propriétaire de l'étang.

Durant le $\mathrm{xix}^{\mathrm{e}}$ siècle, lors de la réglementation des ouvrages d'étangs, l'administration avait eu toutes les difficultés à déterminer des frontières précises entre l'espace de l'étang et les terres. Dans l'impossibilité d'agir avant que la situation soit éclaircie par l'intervention de géomètres, elle se faisait l'écho des contestations des propriétaires de part et d'autre et des litiges récurrents: les procès jalonnent tout le xix ${ }^{\mathrm{e}}$ siècle. Il y est question d'emplacement d'ouvrages, de hauteur du déversoir, des usages reconnus de pacage et d'abreuvage, de surfaces de terre gagnées sur l'eau et contestées par les propriétaires riverains... Comme on ne peut trancher sans la connaissance de la réalité sur le terrain, des géomètres sont dépêchés sur les lieux, puis plus tard des « arpenteurs géographes » qui effectueront le bornage.

Il arrive aussi que, par souci de clarification et de tranquillité - car tant que le bornage d'un étang n'est pas établi, les protestations des riverains ne s'apaisent jamais longtemps -, ce soit le propriétaire de l'étang lui-même qui demande la détermination juridique des contours. Celle-ci ne se définit pas sans enquêtes minutieuses auprès de tous les riverains, et elle fait parfois l'objet, car les points de vue sont différents, d'une longue procédure.

Les faits ne sont pas bien différents aujourd'hui, et le cas de figure se répète: les eaux d'un étang submergent-elles une parcelle cultivée, ou l'agriculteur a-t-il exagérément avancé ses cultures dans l'espace de l'étang qui a du mal à se remplir ? «L'étang est dans ma récolte ! - Non c'est votre récolte qui est dans mon étang! », la joute est habituelle, mais elle s'atténue si un relevé des contours de l'étang « eaux tendues » a été établi. Document exhumé, la querelle s'apaise, mais un moment seulement, car il y a là justement le lieu même de la tension 
entre monde paysan et propriétaires d'étangs, tension elle-même créatrice des liens qui fondent la société brennouse.

De façon générale, ces droits de pacage et d'abreuvage paraissent, aux yeux des propriétaires d'étangs, mal déterminés. Les animaux peuvent pacager en bordure, boire dans l'étang, mais dès lors que les étangs sont très bas, il n'est pas rare de voir les bêtes avancer dans l'étang, ou bien des parties de l'étang, non recouvertes d'eau, labourées et ensemencées. Juste revanche pour ceux qui exploitent les terres : lorsque l'eau, qui a avancé et inondé s'est retirée, alors ce sont les terres qui prennent le dessus.

Et de même que les agriculteurs savent évaluer les hectares qui leur sont ravis par ne serait-ce qu'une dizaine de centimètres de rehaussement du niveau des eaux, de même les hommes d'étangs savent, eux, très vite évaluer selon les étangs, selon les bordures, quelle surface en eau peut être découverte, si le niveau de l'étang est maintenu plus bas de quelques centimètres.

On assiste ainsi à une sorte de va-et-vient, de balancement entre eaux et terres, à l'image d'un rapport de forces qui toujours s'inverse entre propriétaires d'étangs et agriculteurs. L'eau qui inonde, s'étale, recouvre les terres d'où elle chasse les hommes, puis se rétracte. Ces derniers reprennent alors les terres, mais jusqu'à la surface qui n'est pas encore couverte d'eau, « rentrent dans l'étang », pour peu qu'une année soit sèche, et s'aventurent encore. Ce petit jeu est emblématique d'une rivalité dans l'emprise sur l'espace, et de l'équilibre qui parvient à s'installer, qui fait que l'on se supporte et que l'on cohabite tout en se surveillant de près.

Par les étangs, le lien entre deux mondes

La densité des étangs est telle en Brenne que tous les agriculteurs y vivent auprès d'étangs. À un moment ou à un autre, ils en ont subi les débordements, ou ils y ont mené leurs bêtes, mais aussi, ils ont prêté main-forte. Nombreux sont ceux qui aujourd'hui s'occupent d'étangs, soit parce que, fermiers sur les terres qui environnent l'étang, leur aide active est sollicitée, soit parce que propriétaires, gardes ou négociants comptent éventuellement sur les exploitants riverains ou fermiers, ou encore sur les agriculteurs retraités, pour jeter un œil en passant et le surveiller, durant par exemple les opérations délicates de mise en tire puis de mise en pêche. Lorsque l'on n'est pas agriculteur soi-même, le père l'était et, métayer ou fermier, il avait forcément eu affaire aux étangs. Dès lors, une population large d'hommes se voit concernée par les étangs. Toute sa vie aussi, on a côtoyé le maître des domaines et du père métayer, comme ensuite les fils du propriétaire ont pu à leur tour être les «patrons » pour lesquels on a travaillé. 
Il en est ainsi parfois sur plusieurs générations, et l'on ne peut comprendre la nature complexe des liens qui s'établissent autour des étangs aujourd'hui, sans prendre en compte cette donnée essentielle. D'un côté et de l'autre, la force de la relation, issue de cette transmission générationnelle même, est énoncée, sans que pour autant, d'un côté comme de l'autre, on ignore la distance qu'il ne s'agit jamais d'abolir.

Aussi, lorsque le lien est déjà établi et ancien, on ne saurait refuser un service demandé, que l'on soit fermier sur les terres de celui qui demande, exploitant à son compte ou fermier d'un domaine proche de l'étang. Les services ou coups de main rendus sur les étangs, rémunérés ou pas, sont innombrables. Parfois l'agriculteur en vient à s'occuper lui-même plus largement des étangs. Cela arrive tout naturellement, et cela est d'autant plus «normal » que déjà lorsque le père était métayer, il avait à prendre soin de l'étang, assurait la mise en tire, la mise en pêche, la pêche elle-même; cela faisait partie des tâches qu'il avait à accomplir dans le cadre du contrat qui le liait au propriétaire, et, pour avoir toujours suivi et aidé son père, l'agriculteur d'aujourd'hui sait y faire. Ce dernier peut aussi être d'un réseau plus large d'amitié et d'entraide d'agriculteurs et de gardes qui ont la charge de l'exploitation piscicole sur certains étangs; une diffusion de savoir-faire plus élaborés s'opère ainsi, dont profitent les propriétaires exploitants. Le moment venu, l'agriculteur saura trouver la main-d'œuvre nécessaire aux différentes tâches pour la pêche de l'étang à laquelle il participe bien sûr.

Lorsqu'on ne vit pas dans la région et qu'on ne dispose pas d'un garde, on peut aussi demander à l'agriculteur proche de l'étang de s'occuper entièrement de l'étang. À la mort de l'agriculteur, on s'adresse au fils, qui se voit alors appeler à « prendre la relève ». Le fait que les agriculteurs soient sur place, installés non loin des étangs, est d'ailleurs utile pour tous, propriétaires, négociants ou gardes, qui ont souvent de nombreux étangs à surveiller.

Avant même le jour de pêche à laquelle participent nombre d'agriculteurs, la veille de la pêche, les hommes dorment sur la chaussée pour surveiller le niveau des eaux ou chasser les oiseaux; le garde de la propriété, lui-même le plus souvent d'origine paysanne, passe la nuit avec des amis, des parents, des voisins: beaucoup sont agriculteurs.

Ainsi les relations entre agriculture et exploitation des étangs sont fortes, diverses et complexes. Les propriétaires parlent des tolérances accordées de pacage et d'abreuvage des bêtes sur les étangs en compensation des débordements d'étangs, tolérances qui en retour ne sont pas sans avantages aussi pour les propriétaires exploitants piscicoles eux-mêmes. L'« invitation » offerte, de participer aux pêches, est également à leurs yeux comme une contrepartie des 
inconvénients liés aux crues, ou vient en remerciement pour les services rendus, tandis que, de leur côté, les agriculteurs, qui se considèrent eux-mêmes redevables de la tolérance accordée de pacage et d'abreuvage, estiment ne pouvoir refuser de rendre service, ou de participer aux pêches, comme on les y «invite $\gg .^{20}$ Les obligations multiples et réciproques, véritables ou ressenties, donnent une plus grande densité encore au lien.

«On ne refuse pas l'eau. »

Même s'il y a restriction des tolérances dans l'utilisation de l'eau des étangs, il semble difficile à un propriétaire traditionnel de penser que l'accès à l'eau puisse être refusé à des agriculteurs. À la différence des propriétaires récents qui achètent essentiellement en Brenne pour le loisir et n'entrent pas dans les préoccupations locales, les propriétaires exploitants piscicoles, souvent aussi à la tête de domaines anciennement cultivés, ou possédant des fermes encore exploitées, n'ignorent pas l'importance de l'eau en saison d'été pour l'élevage, ni les difficultés causées aux éleveurs par l'assec d'étangs aux abords desquels sont ordinairement tolérées les bêtes. Aussi ont-ils du mal à ne prendre en compte que les impératifs des chasseurs au gibier d'eau sur les étangs, et ils estiment l'abreuvage prioritaire. Et s'ils font sentir aux agriculteurs qu'ils ne doivent pas abuser de la tolérance accordée, par exemple ne pas pomper l'eau comme cela a pu se faire ici et là, s'ils vont parfois jusqu'à menacer de clôturer, la possibilité donnée de profiter de l'eau de l'étang n'est pas remise en cause: «Il faut bien, lorsque l'eau est si rare, leur en laisser l'usage: on ne peut tout de même pas refuser l'eau ! »

La même formulation revient, avec la même véhémence: pas plus entre propriétaires d'étangs que de propriétaire d'étang à agriculteur éleveur, l'eau ne se refuse. Les hommes de Brenne partagent une égale considération pour l'eau.

Mais là encore, comme dans les relations d'étang à étang, le principe éthique énoncé cache de véritables contraintes: parce qu'ils ont éminemment besoin de leurs services et de leur tolérance, les propriétaires d'étangs ne sauraient refuser l'eau aux agriculteurs dont d'une certaine façon ils dépendent à leur tour. ${ }^{21}$

Ainsi à tous les niveaux du social, les hommes sont tenus les uns aux autres. Reconnu par tous, le caractère précieux de l'eau entraîne une gestion de l'eau comme d'un bien rare qui fédère exploitants piscicoles sur les lignes d'étangs et exploitants piscicoles et agricoles autour des étangs. Organisation rationnelle des eaux et survie de l'élevage ne sont possibles que du fait même de ces 
obligations multiples et réciproques dont la complexité apparaît comme en condensé sur les étangs eux-mêmes.

\section{L'étang, un capital symbolique}

Au Portugal, dans la région de Melgaço, seuls ceux qui se disent et que l'on nomme des héritiers possèdent des droits d'eau, et ils tiennent fortement à ce statut privilégié constructif d'une identité prestigieuse. Un système hydraulique complexe se perpétue, plus pour ce qu'il représente à leurs yeux que pour le rapport d'une agriculture que n'est plus nécessaire à personne.

Sur ce dernier point, la situation est comparable en Brenne où prestige et pouvoir sont associés déjà anciennement à la propriété des étangs et à leur exploitation, longtemps activité lucrative, longtemps aussi privilège des seigneurs religieux ou laïques. Et les grandes familles locales tiennent à l'exploitation piscicole également pour des raisons tout autres qu'économiques. La connotation symbolique des étangs, à l'œuvre depuis toujours, se révèlera avec force durant la période révolutionnaire où les paysans s'attaqueront aux étangs pour eux si emblématiques d'une classe sociale.

Aucun document écrit n'atteste de l'existence en Brenne d'un véritable système d'exploitation d'étangs en termes de pisciculture avant le $\mathrm{xv}^{\mathrm{e}}$ siècle. Des recherches récentes ${ }^{22}$ toutefois suggèrent que l'existence de la carpe, espèce courante dans l'est de l'Europe, n'était pas inconnue en France au début du xiiie siècle. À la fin du siècle, l'élevage en était déjà introduit à une échelle large, et la carpe, en quelques décennies, devenue un poisson commun. Dès le début du xiv siècle, l'élevage de cette espèce robuste, tolérant des milieux naturels très divers, en était déjà largement répandu et avait une place de choix dans l'exploitation piscicole.

L'analyse des documents d'archives montre d'ailleurs sans conteste que la production des étangs était, déjà avant le $\mathrm{xv}^{\mathrm{e}}$ siècle, une production rentable à laquelle on accordait la plus grande attention. Et l'on a pu même parler pour la Brenne du $\mathrm{Xv}^{\mathrm{e}}$ siècle d'une véritable «politique des étangs ${ }^{23}$ » mise en œuvre au sein du prieuré local de Loups, à l'époque des prieurs Pierre et Guillaume de Fougères, qui furent aussi un temps abbés de Fongombault.

Des documents d'archives de tous ordres abondent pour les $\mathrm{xv}^{\mathrm{e}}$ et $\mathrm{xvi}^{\mathrm{e}}$ siècles. ${ }^{24}$ Ils nous donnent une mesure sûre de la production des étangs et révèlent un intérêt affirmé pour l'exploitation piscicole, aussi bien de la part des ordres religieux monastiques que des seigneurs et propriétaires laïcs. Ils témoignent clairement aussi d'une conception d'ensemble d'un système d'étangs déjà bien en place et des nombreuses précautions qu'elle suppose, éclairent sur 
les préoccupations et le savoir partagés dans l'aménagement des étangs, l'exigence qu'il impose, et les tentatives enfin, déjà à l'époque, de s'entendre pour une gestion raisonnée qu'illustrent aussi amplement les différents coutumiers locaux qui peu à peu se sont élaborés. Une construction maîtrisée des étangs en vue d'une gestion raisonnée des étangs, une production vite devenue rentable : l'ensemble des documents l'attestent avec certitude. Plus discrètement, ça et là, ils dévoilent déjà aussi le caractère précieux de l'eau des étangs pour le monde paysan alentour.

Dans les siècles suivants, la pisciculture en étangs ne va cesser de prendre de l'ampleur. Selon les historiens, l'économie des étangs, dans la France du

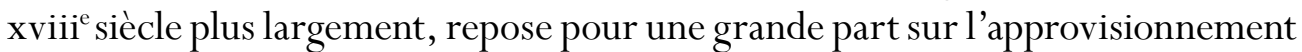
des villes en poissons destinés essentiellement à une élite, friande de ce poisson qui arrive vivant. Car le poisson d'eau douce dont les espèces sélectionnées sont résistantes au point de supporter un long cheminement, est toujours livré en vie, tradition qui demeure jusqu'à nos jours. Et il y aurait bien désormais et de façon générale dans la pisciculture des régions d'étangs et du fait de la consommation urbaine « une activité hautement spéculative..$^{25}$ »

En Brenne, à la fin du $x x^{e}$ siècle puis surtout dès les premières années du $\mathrm{xx}^{\mathrm{e}}$ siècle, avec l'accession plus large à la propriété de familles aristocratiques ou d'origine bourgeoise, les propriétaires qui exploitent leurs étangs s'emploient avec vigueur à développer et moderniser une production piscicole délaissée. L'analyse des écrits de l'époque laisse toutefois aussi paraître un lien à l'étang qui dépasse largement l'intérêt pour sa production.

Ainsi, et pour ne prendre qu'un exemple, un roman publié au début du $\mathrm{xx}^{\mathrm{e}}$ siècle par un écrivain local situe bien les choses. L'étang qu'évoque l'auteur par l'intermédiaire de son personnage, propriétaire en Brenne, n'est pas hérité et vient d'être acquis, mais il est d'emblée inscrit dans la transmission future, aimé déjà pour cela. Le procédé anthropomorphique, lorsque le romancier évoque l'étang, est appuyé et sans détour: l'auteur parle de l'étang comme d'un être vivant pour lequel on éprouve un sentiment, avec lequel s'établit un échange et, le temps passant, une connivence. Au maitre, il semble que son étang lui sourit, de son «serviteur », le maître attend qu'il réponde à ses devoirs et supporte le moment du sacrifice, celui de la pêche qui le défigure, sans en vouloir à son maître dont il accepte l'exigence.

Le devoir est réciproque; si les propriétaires des grandes familles locales expriment, en même temps que leur intérêt pour la pisciculture, un lien affectif sûr à l'étang, ils disent aussi leur devoir par rapport à l'étang qui leur a été transmis et que certains d'entre eux, avec les difficultés de la pisciculture 
aujourd'hui et ses exigences, n'ont plus toujours plaisir à honorer: on s'occupe des étangs parce que l'on en a hérité et parce qu'on se doit de léguer à son tour ses étangs entretenus et productifs. La charge et la responsabilité de la transmission excluent toute légèreté. Perpétuer l'exploitation piscicole est aussi dans cette société se perpétuer soi-même, son nom, sa famille, son existence sociale.

Il y a dans la pisciculture, telle qu'elle est pratiquée au sein d'un monde qui a considérablement changé et devient incertain, le sentiment rassurant d'une continuité, et, dans le lien aux étangs, l'attachement à une tradition que l'on voudrait parvenir à transmettre en même temps que l'on transmettra ses étangs. Mais aujourd'hui les désillusions, les inquiétudes sont si fortes, la pisciculture considérée si menacée, que cela ne se fait pas toujours en toute sérénité. Alors que pour certains propriétaires récents, sans véritable insertion locale, peu encombrés du poids de l'histoire, venus en Brenne pour le plaisir de chasser, pratiquer le cycle de la pisciculture est une distraction: cette façon de voir les choses ne saurait être celle des propriétaires traditionnels.

Pour les membres des vieilles familles locales en l'occurrence, l'étang n'est pas seulement partie du patrimoine hérité et qu'il faut préserver, il est aussi intimement lié à la vie familiale. Qu'on le voit ou pas depuis chez soi, il est, dans la représentation que l'on en a, partie intégrante de la maison familiale, et on l'exprime parfois le plus clairement du monde: «L'étang, pour vous dire, c'est une pièce de la maison. » L'attachement sentimental à l'étang est manifeste. Héritiers de ces familles qui ont agi sur la nature, transformé et modelé le paysage, on continue, lorsqu'on agit sur les étangs à laisser son empreinte dans l'espace, on pérennise celle de ses ascendants. Qu'un ouvrage soit réparé, on grave quelque part la date de la restauration, parfois simple incision; ou encore, elle est sculptée dans le bois de la bonde d'un grand étang, et un cœur lui est associé.

Ainsi, par les étangs et leur agencement, s'exprime l'emprise des familles sur l'espace. L'univers familial s'y projette. On nomme un nouvel étang du nom d'un enfant, ou lorsqu'on n'a pas de descendant direct, du nom de ses nièces ; on le nomme aussi du nom d'un être aimé, celui de l'épouse pour laquelle le sentiment s'inscrit ainsi dans le territoire et par là même se dit au grand jour et s'impose à tous, devenant d'une certaine façon publique.

Mais, lorsque survient le moment de la succession, c'est un ébranlement. Il arrive d'ailleurs que l'on ne partage pas les biens, que l'on préfère garder intact le territoire d'un seul tenant, mais cela n'est pas longtemps possible et le risque est déjà, outre le morcellement, que l'on ne poursuive pas ce qui était auparavant, ou que les habitudes changent, et notamment que d'autres conceptions de la chasse apparaissent sur le territoire. 
Aussi une succession peut-elle être vécue comme un large bouleversement. Énoncer qu'« à chaque succession, le paysage de la Brenne change » n'est pas faux lorsque la propriété a quelque mille hectares. Les familles se font une juste représentation de leur emprise sur le territoire: avec la division des biens qui suit un héritage, ce n'est plus une page mais en effet « des pages d'histoire qui se tournent ». Tant dans cette société, histoire familiale et histoire du territoire se confondent.

Mais si l'étang a été et demeure un capital essentiellement symbolique, et avant tout la marque familiale d'une présence dans l'espace, le signe d'une emprise, si la rentabilité de l'étang n'est finalement pas ce qui prime, il n'en reste pas moins qu'il s'agit, même aux yeux des propriétaires issus des vieilles familles locales, d'en tirer si possible un bon revenu - cela a longtemps été le cas, et l'est encore parfois -, sûrement de ne pas perdre d'argent, au minimum de rentrer dans ses frais pour perpétuer l'exploitation des étangs. Mais aujourd'hui, les difficultés s'accumulent: à la sécheresse qui s'aggrave, s'ajoute la présence sur le territoire de nouvelles espèces qui détruisent la végétation de l'étang et ses ouvrages. Il devient difficile, dans un tel contexte, et pour parvenir à maintenir l'exploitation qui n'est plus rentable, de résister à louer ses étangs pour la chasse, surtout lorsque la location dans cette région préservée et sur des étangs cachés au sein des domaines peut atteindre des prix sans commune mesure avec ce que rapporte la pisciculture. Certains étangs sont loués depuis longtemps, la chasse semble être alors la condition pour continuer à pratiquer la pisciculture et l'entretien par là même de l'étang auquel on lie son propre destin.

Dès lors, rester chez soi dans son domaine où l'on a tout fait pendant des générations pour ne pas avoir d'enclaves, garder les étangs exclusivement pour soi, continuer à les exploiter sans les louer, deviennent des privilèges incommensurables, auxquels seuls ont accès encore quelques très rares propriétaires de grandes familles de Brenne, et certainement les propriétaires récents et à venir, disposant de grandes fortunes leur permettant d'acheter en Brenne de vastes espaces, mais, eux, pour le plaisir exclusif d'y chasser sur les eaux de leurs étangs ou dans leurs bois.

\section{Conclusion: les priorités qu'une société se donne}

Ainsi à un moment ou à un autre de son histoire, une société peut être amenée à exercer une pression telle sur ses ressources qu'elles en deviennent rares et sont bien considérées telles: pour l'eau, un système rigoureux de gestion est aussitôt mis en place qui peut persister, alors que les conditions socio-économiques ont profondément et depuis longtemps changé. Au Portugal, bien loin 
de la Brenne dont a été privilégié l'exemple, l'eau est toujours abondante. Mais, consacrée en saison d'été à une production que l'on peut désormais aujourd'hui se procurer aisément par ailleurs, elle reste précieuse, et le système, avec sa stricte organisation, perdure dans toute sa complexité pour d'autres raisons auxquelles les hommes demeurent tout autant attachés. En cela on ne peut parler de l'inertie d'un système hydraulique originel dont les hommes auraient perdu le sens (Berque, 1955:157-159 ; Bédoucha, 1987:43-46) et auquel ils se contraindraient sans le comprendre: le système est au contraire surinvesti de valeurs fortement sensibles et d'enjeux socio-politiques locaux. Les hommes se plient certes à un ordre ancien mais pour d'autres raisons toutes actuelles.

Alors que dans de nombreuses sociétés hydrauliques, ${ }^{26}$ la société se projette dans l'organisation de la distribution de l'eau qui dès lors en vient à refléter l'organisation sociale, c'est l'inverse qui, dans la société brennouse, semble bien s'être produit. Le système mis en place il y a bien longtemps au sein d'une même propriété seigneuriale, qu'elle soit laïque ou religieuse, pour l'organisation de la gestion des eaux de ses étangs solidaires, et qui n’a cessé de s'élargir et de s'affiner, agit sur les hommes. Les propriétaires ont changé, les grandes propriétés se sont morcelées ; très nombreux, les étangs toujours reliés les uns aux autres sont en de nombreuses mains. Mais les grandes familles qui semblent toujours associer leur sort à celui des étangs, dans des conditions aujourd'hui difficiles s'astreignent à leur exploitation, non pour survivre économiquement puisqu'elles n'ont jamais véritablement vécu de leurs étangs mais pour que les étangs survivent en ce qu'ils représentent symboliquement pour elles.

La dépendance des étangs entraîne par la force des choses celle des hommes, et ils le tolèrent, du moins tant qu'ils considéreront l'exploitation piscicole comme essentielle à leurs yeux, et pour cela même l'eau comme une ressource précieuse, ce qu'elle demeure pour le monde rural alentour.

Recebido em 22/07/2011

Aprovado em 04/08/2011

Les travaux de Geneviève Bédoucha, directeur de recherche au CNRS en anthropologie sociale, portent sur les rapports entre gestion de l'eau et société. Elle a longtemps mené des enquêtes en zone aride ou semi-aride sans l'aire tribale du monde arabe, notamment en Tunisie et au Yémen, où elle a effectué des analyses comparatives, avant de s'intéresser aux zones humides continentales. 


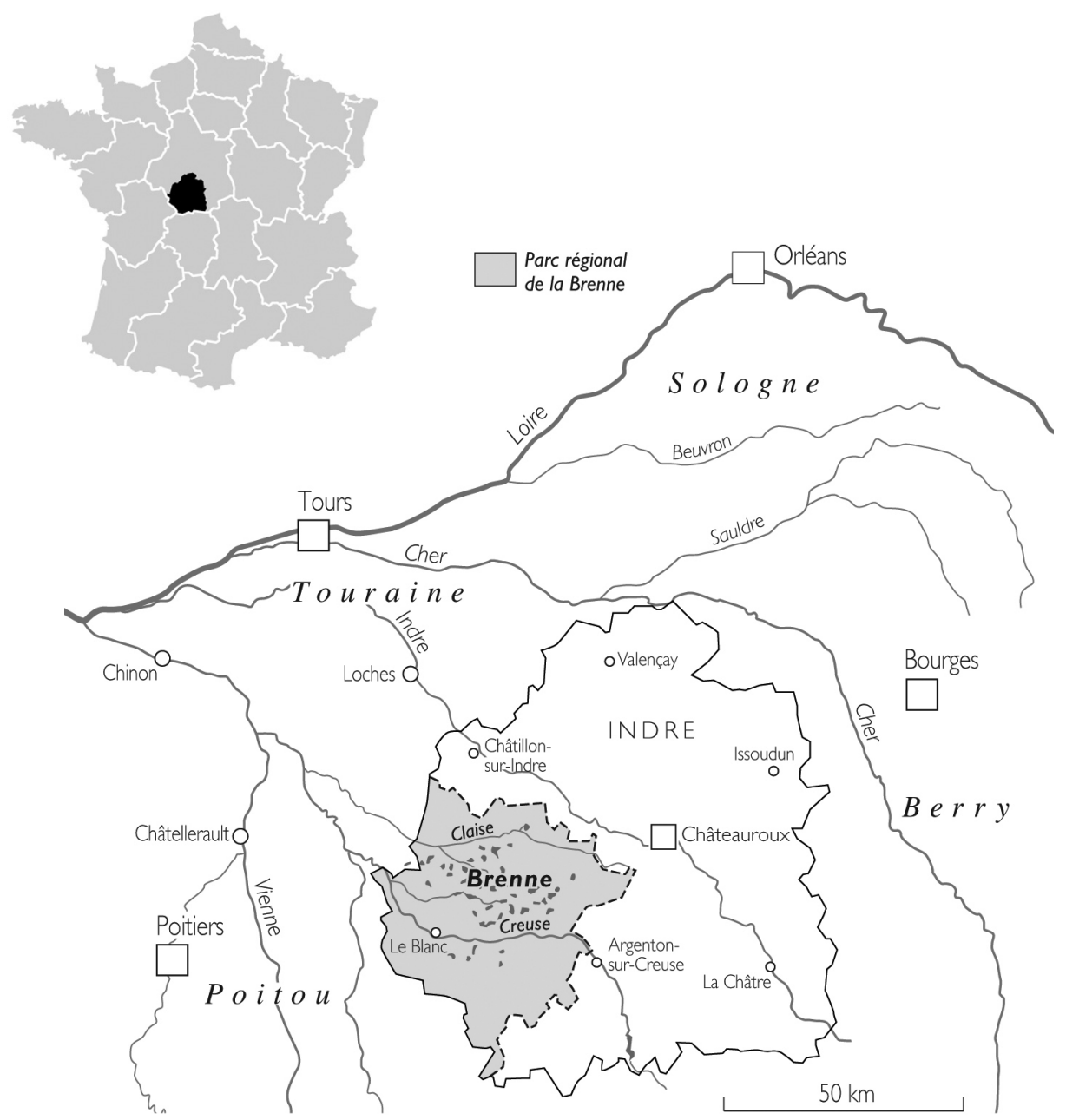

Figure 1: La Brenne, au coeur de la France 


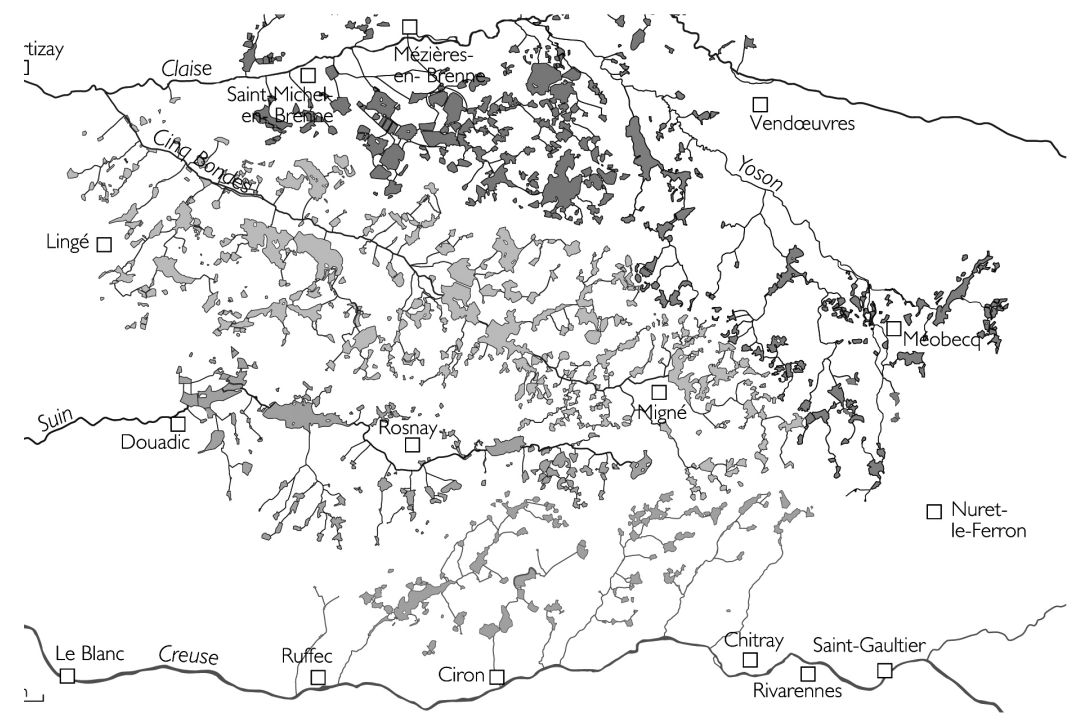

Figure 2: Le réseau hydrographique d'ensemble

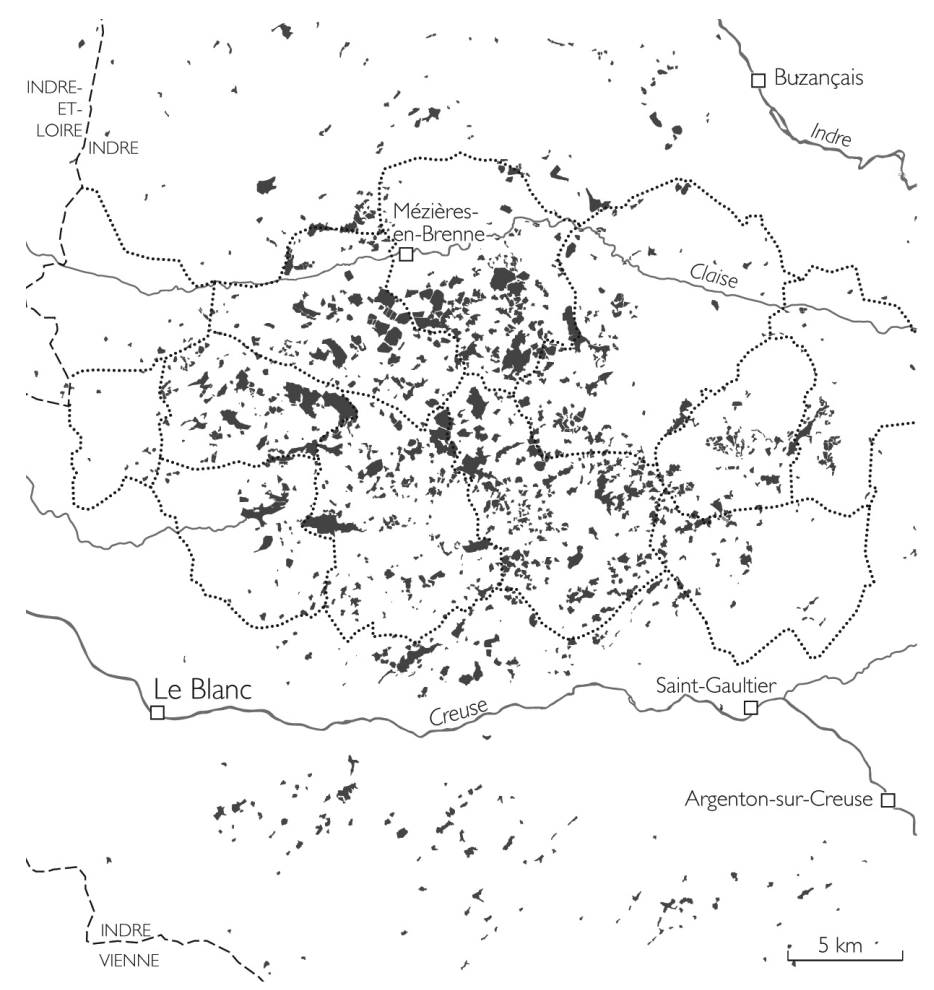

Figure 3: Une constellation d'étangs 


\section{Notas}

1. Berque 1955, Fernea 1970, Bédoucha 1987, Aubriot 2004.

2. Ainsi et par exemple, Aguilera-Klink, Pérez-Moriana, Sánchez-García, 2000; Buchs, 2009.

3. Le terme est emprunté à O. Ribeiro (1987) par F. Wateau (2004) et sans doute ici peut-on l'entendre dans les deux acceptions possibles, abondance de d'eau et prospérité de ceux qui en bénéficient.

4. Le gaspillage d'eau constaté par ailleurs de nos jours n'induit en rien qu'il en ait été de même autrefois. La gestion au XVII ${ }^{e}$ siècle a pu être plus rigoureuse.

5. La cartographie a été réalisée en collaboration avec Anne Le Fur de l'AFDEC à Paris.

6. Une enquête de terrain en Brenne a eu lieu de façon continue de septembre 1998 à mai 1999, elle s'est ensuite prolongée sur plusieurs années jusqu'en 2008 au cours de nombreux séjours d'un à deux mois à différentes saisons.

7. Mais le chiffres varient encore selon l'espace pris en compte.

8. Pour une analyse plus approfondie, je renverrai à Bédoucha, 2011.

9. Lorsque je parlerai d'hommes d'étangs, il s'agira de tous ceux qui s'occupent des étangs et de la gestion des eaux, qu'ils soient propriétaires exploitants, négociants-pisciculteurs, gardes, ou même simplement agriculteurs à qui est parfois confié le soin des étangs.

10. Hormis ceux dont l'eau part directement dans un ruisseau. Il existe par ailleurs en Brenne très peu de fossés de dérivation connus dans la Dombes sous le nom de «rivières de ceinture » ou «rivières de détourne », qui dévient en effet l'arrivée de l'eau amenée plus loin et donnent plus d'indépendance aux étangs.

11. On notera qu'il s'agit du même nom mais au féminin.

12. Sorte d'épuisette sans manche.

13. Archives départementales de l'Indre, série $S$.

14. Alevins de carpe.

15. Manceron, 2005:119.

16. Ibid : 158.

17. On a manqué de paille pour les litières jusqu'après la Seconde Guerre mondiale. La Champagne berrichone toute proche a alors développé la céréaliculture, suffisamment pour que les besoins en paille aient pu être couverts localement, on se souvient pour cette époque d'«acheteurs de paille» dont l'existence atteste de l'ampleur des besoins.

18. 15 centimètres de niveau peuvent prendre 50 mètres de prairie de chaque côté de l'étang. Cela finit par faire 2-3 hectares de gagnés sur la prairie.

19. Il est évalué à 5000 litres par jour pour un troupeau de trente bêtes. Et sur un petit étang, pour peu que l'été soit sec, « les bêtes pourraient bien mettre l'étang à nu ! », dit un agriculteur lui-même. 
20. Cette complexité de la notion d'invitation est analysée dans tous ses aspects par G. Bédoucha, 2011.

21. Sans compter que les enjeux municipaux ont dû sans doute avoir leur place. Maires des communes, les membres de ses grandes familles locales ont aussi certainement été attentifs au vote dans le monde paysan, autre type de dépendance.

22. Benarrous et Marinval, 2006.

23. Bascher, 1980:38.

24. Archives départementales de l'Indre, séries E, G, H.

25. Abad, 2006:14.

26. J'entends par société hydraulique une société structurée en lien étroit avec la gestion de l'eau qui y tient une place essentielle. 


\section{Références Bibliographiques}

ABAD, Reynald. 2006. La conjuration contre les carpes. Enquête sur les origines du dessèchement des étangs du 14 frimaire an II. Paris: Fayard.

AGUILERA-KLINK, Federico, PÉREZ-MORIANA, Eduardo et SANCHEZ-GARCIA, Juan. 2000. "The Social Construction of Scarcity: the Case of Water in Tenerife (Canary Islands)", Ecological Economics, 34 (2):233-245.

AUBRIOT, Olivia. 2004. L'eau, miroir d'une société. Irrigation paysanne au Népal central Paris: CNRS Editions, collection Monde Indien, Sciences sociales $15^{\mathrm{e}}-21^{\mathrm{e}}$ siècle.

BASCHER, Dom Jean de. 1980. Le prieuré de Loups, première fondation de l'Abbaye de Fontgombault et les seigneurs de Brenne. 1096-1791. Châteauroux: Imprimerie Laboureur.

BÉDOUCHA, Geneviève. 1987. L'eau l'amie du puissant, une communauté oasienne de l'extrême Sud tunisien. Paris: Éditions des archives contemporaines (Collection Ordres sociaux). . 2011. Les liens de l'eau. En Brenne, une société autour de ses étangs. Paris: Éditions de la Maison des sciences de l'homme/ Éditions Quae (Collection Natures sociales).

BENARROUS, Renaud et MARINVAL, Marie-Christine. 2006. « La carpe, Cyprinus carpio, cette orientale qui séduit l'Occident au Moyen Âge ». In DEREX, J.-M. (éd.). La production des étangs du Moyen Âge à l'époque contemporaine. Actes de la Journée d'étude 2005 du Groupe d'Histoire des Zones Humides. pp. 9-22.

BERQUE, Jacques. 1955. Structures sociales du Haut Atlas. Presses universitaires de France. BUCHS, Arnaud. 2009. «Crise de l'eau et construction sociale de la pénurie. L'exemple de l'usage de l'eau à Almeria (Andalousie) ». In Forum de la régulation 2009: Les défis analytiques et pratiques posés en économie par les crises et les problèmes de régulation: terrains et méthodes en question. 1-2 décembre 2009, Paris.

FERNEA, Robert-Alan. 1970. Shaykh and Effendi: Changing Patterns of Authority among the El Shabana of Southern Iraq. Cambridge, Massachusets: Harvard University Press (Harvard Middle Eastern Studies, 14).

HONEGGER, Anne, et BRAVARD, Jean-Paul (éds). 2005. "La pénurie d'eau: donnée naturelle ou question sociale ». Géocarrefour, 80 (4):3-4.

JACOB-ROUSSEAU, Nicolas. 2005. Aspects de la pénurie hydrique et de sa gestion dans la Cévenne vivaraise (I : le xix ${ }^{\mathrm{e}}$ siècle), in Honneger et Bracard (éds). Géocarrefour, 80 (4), [En ligne], mis en ligne le 01 juin 2009. URL: http://geocarrefour.revues.org/1278. 
LABBAL, Valérie. 2001. 'Travail de la terre, travail de la pierre'. Des modes de mise en valeur des milieux arides par les sociétés himalayennes. L'exemple du Ladakh. Thèse de doctorat, Université Aix-Marseille 1, Université de Provence.

LATOUR, Bruno. 1993. We have never been modern. Cambridge, Massachusets: Harvard University Press.

MANCERON, Vanessa, 2005. Une terre en partage. Liens et rivalités dans une société rurale. Paris: Éditions de la Maison des sciences de l'homme (Collection Ethnologie de la France).

RIBEIRO, Orlando. 1987. Portugal. O Mediterrâneo e o Atlântico. Esboços de relações geográficas. 5ème édition. Lisbonne: Livraria Sá da Costa Editora.

TROTTIER, Julie. 2008. "Water crises: political construction or physical reality? “. Contemporary Politics, 14 (2):197-214.

WATEAU, Fabienne. 2002. Partager l'eau: irrigation et conflits au nord-ouest du Portugal. Paris: CNRS Éditions/Éditions de la Maison des sciences de l'homme).

. 2008. La canne à mesurer l'eau, http://videotheque.cnrs.fr/index.

php? urlaction $=$ doc\&id_doc $=1809$ 


\section{Resumo:}

Este artigo pretende explorar o paradoxo da existência de uma gestão rigorosa da água em diferentes lugares do mundo onde a água é, no entanto, abundante. A análise focaliza a região de Brenne, no centro da França, onde milhares de tanques existem hoje em milhares de hectares. A análise do sistema de exploração dos tanques, inaugurado na Idade Média, demonstra que a água nesta zona úmida é valorizada como um recurso precioso. A autora argumenta que a importância simbólica dos tanques atravessou os séculos e que claramente os proprietários desejam manter os tanques de piscicultura por outras razões além das econômicas. Uma vez que uma melhor gestão do sistema em sua totalidade exige desperdiçar o mínimo de água possível, eles têm que trabalhar em conjunto. A água dos tanques também é valiosa para os agricultores que, na estação quente, dela dispõem como um complemento fundamental para dar de beber ao gado. A preciosidade da água é, portanto, de interesse comum para todos: ela organiza toda a sociedade.

Palavras-chave: Escassez ou abundância de água, gestão da água, tanques, piscicultura, Brenne

\section{Résumé}

Est interprété le paradoxe d'une gestion rigoureuse de l'eau en différents lieux du monde où l'eau se trouve pourtant en abondance. L'analyse porte plus particulièrement sur un système d'exploitation d'étangs mis en place déjà au Moyen-Âge en Brenne, région du cour de la France, où existent de nos jours des étangs par milliers répartis sur plusieurs milliers d'hectares, et montre qu'en cette zone humide l'eau est bien considérée comme une ressource précieuse. L'importance symbolique des étangs a traversé les siècles, et les propriétaires exploitants tiennent à perpétuer la pisciculture pour des raisons tout autres qu' économiques. Mais une meilleure gestion du système d'ensemble leur impose de s'entendre afin de gaspiller le moins d'eau possible. L'eau des étangs s'avère également précieuse pour les agriculteurs éleveurs alentour, qui, en saison chaude y trouvent un appoint capital pour l'abreuvage des bêtes. Dès lors, le caractère précieux de l'eau pour les uns et les autres structure l'ensemble de la société.

Mots clefs: Rareté ou abondance de l'eau, gestion de l'eau, étangs, pisciculture, Brenne 